\title{
Kinetic theory of two dimensional point vortices from a BBGKY-like hierarchy
}

\author{
Pierre-Henri Chavanis
}

November 30, 2018

\author{
Laboratoire de Physique Théorique (CNRS UMR 5152), \\ Université Paul Sabatier, \\ 118, route de Narbonne, 31062 Toulouse Cedex 4, France \\ E-mail: chavanis@irsamc.ups-tlse.fr
}

\begin{abstract}
Starting from the Liouville equation, we derive the exact hierarchy of equations satisfied by the reduced distribution functions of the single species point vortex gas in two dimensions. Considering an expansion of the solutions in powers of $1 / N$ (where $N$ is the number of vortices) in a proper thermodynamic limit $N \rightarrow+\infty$, and neglecting some collective effects, we derive a kinetic equation satisfied by the smooth vorticity field which is valid at order $O(1 / N)$. This equation was obtained previously [P.H. Chavanis, Phys. Rev. E, 64, 026309 (2001)] from a more abstract projection operator formalism. If we consider axisymmetric flows and make a markovian approximation, we obtain a simpler kinetic equation which can be studied in great detail. We discuss the properties of these kinetic equations in regard to the $H$-theorem and the convergence (or not) towards the statistical equilibrium state. We also study the growth of correlations by explicitly calculating the time evolution of the two-body correlation function in the linear regime. In a second part of the paper, we consider the relaxation of a test vortex in a bath of field vortices and obtain the Fokker-Planck equation by directly calculating the second (diffusion) and first (drift) moments of the increment of position of the test vortex. A specificity of our approach is to obtain general equations, with a clear physical meaning, that are valid for flows that are not necessarily axisymmetric and that take into account non-Markovian effects. A limitations of our approach, however, is that it ignores collective effects.
\end{abstract}




\section{Introduction}

Several authors have wondered whether fluid turbulence could be described in terms of statistical mechanics [1]. Three dimensional turbulence has been attacked by different methods [2, 3, 4, 5] inspired by statistical mechanics and kinetic theories. Some progress has also been made in the simpler case of two dimensional turbulence (see reviews in [6, 7, 8]). Two dimensional turbulence is not just academic but is relevant to describe geophysical and astrophysical flows. Two dimensional flows are characterized by the spontaneous formation of large-scale vortices that dominate the dynamics [9, 10]. The most famous example is Jupiter's great red spot, a huge vortex persisting for more than three centuries in a turbulent shear layer between two zonal jets in the southern hemisphere of the planet [11]. Other examples of this self-organization are the cyclones and anticyclones in the earth atmosphere, the jets in the oceans like the gulf stream or the intense jets on Jupiter [12]. As a first step to tackle the problem, it can be of interest to study the dynamics of a system of $N$ point vortices on a plane [13]. Each vortex produces a velocity field that moves the other vortices in a self-consistent manner. The velocity created by a vortex decreases like $1 / r$ which is similar to the Coulombian or Newtonian interaction in two dimensions. Therefore, the interaction between point vortices is long-range, like the interaction between stars in a galaxy or between electric charges in a plasma. Note, however, that point vortices produce a velocity while material particles produce a force (acceleration). Apart from this (important) difference, the point vortex gas has a Hamiltonian structure [14] and we can try to apply the methods of statistical mechanics and kinetic theory to that system. Therefore, the $N$-vortex problem [13] is of fundamental interest in statistical mechanics and kinetic theory. It provides a physical example of systems with long-range interactions, whose dynamics and thermodynamics are actively studied at present [15].

The statistical mechanics of 2D point vortices was first considered by Onsager [16] in a seminal paper. He showed that statistical equilibrium states with sufficiently large energies have negative temperatures. For such states, like-sign vortices have the tendency to group themselves and form clusters. If the circulations of all the point vortices have the same sign, the equilibrium state is a large-scale vortex (supervortex) similar to vortices observed in geophysical and astrophysical flows. When the point vortices have positive and negative circulations, the equilibrium state is generically a dipole made of a cluster of positive vortices and a cluster of negative vortices. The pioneering work of Onsager was pursued by Joyce \& Montgomery [17] and Lundgren \& Pointin [18], using a mean field approximation. Using a combinatorial analysis, Joyce \& Montgomery introduced an entropy for the point vortex gas which is similar to the Boltzmann entropy for material particles. The statistical equilibrium state (most probable) is obtained by maximizing this Boltzmann entropy while conserving all the constraints imposed by the dynamics (total number $N$ of point vortices and energy $E$, as well as angular momentum $L$ and impulse $\mathbf{P}$ for domains with a special symmetry). For point vortices with equal circulation $\gamma$, the smooth vorticity field is given by the Boltzmann distribution $\omega(\mathbf{r})=A e^{-\beta \gamma \psi(\mathbf{r})}$, where the potential is played by the stream function $\psi(\mathbf{r})$. Using $\omega=-\Delta \psi$, the stream-function is then determined by the Boltzmann-Poisson equation. Lundgren \& Pointin started from the exact equilibrium hierarchy of equations satisfied by the reduced distribution functions $P_{j}\left(\mathbf{r}_{1}, \ldots, \mathbf{r}_{j}\right)$ of the point vortex gas and, by neglecting all the correlations between point vortices, derived a differential equation determining the equilibrium distribution of the one-body distribution function $P_{1}\left(\mathbf{r}_{1}\right)$. Using the fact that $\omega(\mathbf{r})=N \gamma P_{1}(\mathbf{r})$, the mean field equation derived by Lundgren $\&$ Pointin coincides with the Boltzmann-Poisson equation derived by Joyce \& Montgomery. In a mathematical work, Caglioti et al. [19] showed rigorously that the mean field approximation is exact in a proper thermodynamic limit $N \rightarrow+\infty$ such that $\gamma \sim 1 / N, E \sim 1, \beta \sim N$ and $V \sim 1$ (where $V$ is the area of the domain). In that limit the $N$-body distribution at statistical 
equilibrium is a product $P_{N}\left(\mathbf{r}_{1}, \ldots \mathbf{r}_{N}\right)=P_{1}\left(\mathbf{r}_{1}\right) \ldots P_{1}\left(\mathbf{r}_{N}\right)$ of $N$ one-body distributions that are solution of the Boltzmann-Poisson equation. This statistical equilibrium state is expected to be achieved for $t \rightarrow+\infty$. We stress, however, that the statistical theory is based on the assumption that "at statistical equilibrium, all accessible microstates are equiprobable". This is essentially a postulate, so there is no guarantee that the point vortex gas will reach a statistical equilibrium state of the form described above (based on the microcanonical distribution). In order to determine the timescale of the relaxation of the smooth vorticity field $\omega(\mathbf{r}, t)$, and in order to establish whether (or not) the system will truly relax towards Boltzmann statistical equilibrium, we must develop a kinetic theory of point vortices.

A kinetic theory was developed by Dubin \& O'Neil [20] in the case of a non neutral plasma confined by a strong magnetic field, a system isomorphic to the point vortex gas. They started from the Klimontovich equation and used a quasilinear approximation to determine the current of the smooth density due to discrete interactions between point vortices. They considered an axisymmetric evolution of the system and, in the course of their derivation, made a Markov approximation assuming that the two-body correlation function relaxes on a timescale that is much shorter than the timescale on which the smooth density field changes (this is the counterpart of the Bogoliubov hypothesis in plasma physics). They obtained a closed expression of the current, see Eq. (11) of [20], taking into account "collective effects" between the particles. These collective effects are similar to those giving rise to the Debye shielding in plasma physics in the Lenard-Balescu approach [21, 22]. In plasma physics, they take into account the fact that a charge is surrounded by a polarization cloud of opposite charges. In the case of point vortices, their physical interpretation and their consequence is more difficult to establish.

A kinetic theory of point vortices was carried out independently by Chavanis [23], using an analogy with the kinetic theory developed for stellar systems. He started from the Liouville equation and used the projection operator formalism of Willis \& Picard [24] to derive a kinetic equation for the smooth vorticity distribution $\omega(\mathbf{r}, t)$. By this method, he obtained a kinetic equation, see Eq. (128) of [23], that is valid for flows with arbitrary symmetry (non necessarily axisymmetric) and taking into account memory effects. This is the counterpart of the generalized Landau equation in stellar dynamics derived by Kandrup [25] using the same formalism, see Eq. (42) of [25]. If we restrict ourselves to axisymmetric flows and make a Markovian approximation, this leads to a simplified kinetic equation, see Eq. (133) of [23], which coincides with the equation obtained by Dubin \& O'Neil [20] when collective terms are ignored. In a sense, the simplified kinetic equation (133) obtained by Chavanis [23] is the counterpart of the Landau [26] equation in plasma physics while the more general equation (11) obtained by Dubin \& O'Neil [20] is the counterpart of the Lenard-Balescu equation [21, 22]. Finally, the general non-Markovian equation (128) of [23] is related to the Master equations discussed by Prigogine [27] in plasma physics. Therefore, there are many interesting analogies between plasma physics, stellar dynamics and vortex dynamics. In these analogies, the position $\mathbf{r}$ of the point vortices plays the role of the velocity $\mathbf{v}$ of the particles in a plasma or in a stellar system, and the angular momentum $L=\int \omega r^{2} d \mathbf{r}$ plays the role of the kinetic energy $K=\frac{1}{2} \int f v^{2} d \mathbf{v}$. Chavanis [23] also considered the evolution of a test vortex in a bath of field vortices with fixed distribution (e.g. a thermal bath at statistical equilibrium) and used the projection operator formalism to derive a Fokker-Planck equation for the evolution of the one-body distribution of the test vortex $P(r, t)$ in the bath. This equation involves a term of diffusion and a term of drift that are both position dependent. For a thermal bath, i.e. when the field vortices are at statistical equilibrium, the diffusion coefficient and the drift coefficient (mobility) are related to each other by a sort of Einstein relation involving a negative temperature (in cases of physical interest). The resulting Fokker-Planck equation, see Eq. (115) of [23], is the counterpart of the Kramers-Chandrasekhar equation, see Eq. (10) of [28], in stellar dynamics. 
The projection operator formalism which is at the basis of the above-mentioned kinetic theory is very abstract and it is not clear to understand which approximations have been made in the course of the derivation. In this paper, we present an alternative derivation of the kinetic equations obtained in 23 , which is more transparent. We start from the exact out-ofequilibrium BBGKY-like hierarchy of equations satisfied by the reduced distribution functions $P_{j}\left(\mathbf{r}_{1}, \ldots, \mathbf{r}_{j}, t\right)$ of the point vortex gas and consider an expansion of the solutions in powers of $1 / N$ in a proper thermodynamic limit $N \rightarrow+\infty$. The kinetic equations obtained in 23$]$ are recovered at the order $O(1 / N)$. The idea of using a BBGKY-like hierarchy to derive the kinetic equations of [23] was given in [29]. This derivation has the advantage of being much simpler than the previous one and shows clearly the domain of validity of the kinetic equations. It also shows how collective terms can be included in the calculations. However, we shall not try to evaluate these terms in the present paper because they require heavy calculations in the complex plane similar to those performed in plasma physics to derive the Lenard-Balescu equation from the BBGKY hierarchy. We hope to come to this problem in a future work.

The paper is organized as follow. In Sec. 2, we consider the statistical equilibrium state. We recall the equilibrium hierarchy derived by Lundgren \& Pointin [18] and the proper thermodynamic limit for the point vortex gas. For $N \rightarrow+\infty$, the two-body correlation function vanishes so that the mean field approximation is exact in that limit. We derive the differential equation satisfied by the smooth vorticity field. For the usual potential of interaction between point vortices, it reduces to the Boltzmann-Poisson equation. We go beyond the mean field approximation and determine the exact integrodifferential equation satisfied by the two-body correlation function at order $O(1 / N)$. In Sec. 3, we consider the out-of-equilibrium problem and derive the BBGKY-like hierarchy satisfied by the reduced distribution functions of the single species point vortex gas. We close the hierarchy by considering an expansion of the solutions in powers of $1 / N$ in the proper thermodynamic limit $N \rightarrow+\infty$ (Sec. 3.1). To leading order in $N \rightarrow+\infty$, we find that the smooth vorticity field satisfies the $2 \mathrm{D}$ Euler equation. This is the counterpart of the Vlasov equation in plasma physics and stellar dynamics. At order $O(1 / N)$, we obtain an exact system of coupled equations taking into account "distant collisions" between point vortices. If we neglect collective effects, we can obtain an explicit kinetic equation that is valid for flows that are not necessarily axisymmetric and that takes into account non-markovian effects. It is valid on a timescale $\sim N t_{D}$. We simplify this equation by considering axisymmetric flows and arguing that markovian effects can be neglected for $N \rightarrow+\infty$ (Sec. 3.2). We discuss the properties of these kinetic equations in regard to the $H$-theorem and the convergence (or not) towards the statistical equilibrium state (Sec. 3.5). We also study the growth of correlations by explicitly calculating the time evolution of the two-body correlation function in the linear regime (Sec. 3.6). In Sec. 4, we show that the above-mentioned kinetic equations can also be obtained from a quasilinear theory starting from the Klimontovich equation. This is the approach considered by Dubin \& O'Neil [20] for axisymmetric flows. We show how it can be generalized to arbitrary flows when collective effects are neglected. We also stress the connection with the quasilinear theory of the 2D Euler-Poisson system developed by Chavanis [30] to describe the process of violent relaxation in the collisionless regime [31, 32, 33, 34]. Finally, in Sec. 5, we consider the relaxation of a test vortex in a bath of field vortices at equilibrium and obtain the Fokker-Planck equation by directly calculating the second (diffusion) and first (drift) moments of the increment of position of the test vortex. We obtain general expressions, with a clear physical meaning, that are valid for flows that are not necessarily axisymmetric and that take into account non-Markovian effects. We simplify them in the case of axisymmetric flows and recover by a direct calculation the Fokker-Planck equation that was obtained in [23] from the more formal projection operator formalism. We also indicate how the results can be generalized when collective effects are taken into account in the problem. 


\section{The statistical equilibrium state}

To set the notations and show the connection with the kinetic theory developed in the next section based on a BBGKY-like hierarchy, we first derive the differential equation satisfied by the smooth vorticity profile at statistical equilibrium from an equilibrium hierarchy [18].

\subsection{The equilibrium hierarchy}

The exact vorticity field of a gas of point vortices with equal circulation $\gamma$ is given by

$$
\omega_{d}(\mathbf{r}, t)=\sum_{i=1}^{N} \gamma \delta\left(\mathbf{r}-\mathbf{r}_{i}(t)\right),
$$

where $\mathbf{r}_{i}(t)$ is the position of the $i$-th point vortex at time $t$. The dynamical evolution of the point vortices is governed by the Hamilton equations

$$
\begin{gathered}
\gamma \frac{d x_{i}}{d t}=\frac{\partial H}{\partial y_{i}}, \quad \gamma \frac{d y_{i}}{d t}=-\frac{\partial H}{\partial x_{i}}, \\
H=\gamma^{2} \sum_{i<j} u\left(\left|\mathbf{r}_{i}-\mathbf{r}_{j}\right|\right),
\end{gathered}
$$

where the positions $(x, y)$ of the point vortices are canonically conjugate [14. For simplicity, we present the results in an unbounded domain where the potential of interaction depends only on the absolute distance between point vortices, but we stress that most of our results can be extended to bounded domains by using the generalized Green function of Lin [35] satisfying the reciprocity property $u\left(\mathbf{r}_{i}, \mathbf{r}_{j}\right)=u\left(\mathbf{r}_{j}, \mathbf{r}_{i}\right)$. The $N$-vortex distribution function satisfies the Liouville equation

$$
\frac{\partial P_{N}}{\partial t}+\sum_{i=1}^{N} \mathbf{V}_{i} \frac{\partial P_{N}}{\partial \mathbf{r}_{i}}=0,
$$

expressing the conservation of the probability density $P_{N}\left(\mathbf{r}_{1}, \ldots, \mathbf{r}_{N}, t\right)$ that the first point vortex is in $\mathbf{r}_{1}$, the second in $\mathbf{r}_{2}$ etc., at time $t$ and where $\mathbf{V}_{i}$ is the total velocity of point vortex $i$. It is created by the other vortices, so that

$$
\mathbf{V}_{i}=\sum_{j \neq i} \mathbf{V}(j \rightarrow i),
$$

where

$$
\mathbf{V}(j \rightarrow i)=-\gamma \mathbf{z} \times \frac{\partial u_{i j}}{\partial \mathbf{r}_{i}},
$$

is the velocity created by point vortex $j$ on point vortex $i$. We shall essentially consider the standard potential of interaction $u_{i j}=-(1 / 2 \pi) \ln \left|\mathbf{r}_{i}-\mathbf{r}_{j}\right|$ but we leave the function $u\left(\left|\mathbf{r}_{i}-\mathbf{r}_{j}\right|\right)$ as general as possible in order to describe different models like, e.g., the quasi-geostrophic model. Any function of the constants of motion of the Hamiltonian dynamics (energy $E=H$, angular momentum $L=\gamma \sum_{i} r_{i}^{2}$ if the domain has rotational symmetry and impulse $\mathbf{P}=\gamma \sum_{i} \mathbf{r}_{i}$ if the domain has translational symmetries) is a stationary solution of Eq. (3). For brevity, we shall only consider the conservation of energy (the case of an infinite domain with conservation of angular momentum is treated in [18]). The basic postulate of statistical mechanics states that, 
at equilibrium, all microscopic configurations that are accessible (i.e. that have the correct value of energy) are equiprobable. There is no guarantee that the dynamics will lead the system to that "uniform" state because we could imagine that some regions of the $2 N$-dimensional phase space could be more probable than others. However, if we accept this postulate, the equilibrium $N$-body distribution is given by the microcanonical distribution

$$
P_{N}\left(\mathbf{r}_{1}, \ldots, \mathbf{r}_{N}\right)=\frac{1}{g(E)} \delta\left[E-H\left(\mathbf{r}_{1}, \ldots, \mathbf{r}_{N}\right)\right]
$$

Using the normalization condition $\int P_{N} \prod_{i} d \mathbf{r}_{i}=1$, we deduce that the density of states with energy $E$ is given by

$$
g(E)=\int \delta\left[E-H\left(\mathbf{r}_{1}, \ldots, \mathbf{r}_{N}\right)\right] \prod_{i} d \mathbf{r}_{i}
$$

The microcanonical entropy of the system is defined by $S(E)=\ln g(E)$ and the microcanonical temperature by $1 / T(E)=\partial S / \partial E$ (we take the Boltzmann constant $k_{B}=1$ ). We introduce the reduced probability distributions

$$
P_{j}\left(\mathbf{r}_{1}, \ldots, \mathbf{r}_{j}\right)=\int P_{N}\left(\mathbf{r}_{1}, \ldots, \mathbf{r}_{N}\right) d \mathbf{r}_{j+1} \ldots d \mathbf{r}_{N}
$$

For identical particles, the smooth (average) vorticity field is related to the one-body distribution function by

$$
\omega(\mathbf{r})=\left\langle\sum_{i=1}^{N} \gamma \delta\left(\mathbf{r}-\mathbf{r}_{i}\right)\right\rangle=N \gamma P_{1}(\mathbf{r})
$$

Note that the vorticity field is proportional to the density of point vortices: $\omega(\mathbf{r})=\gamma n(\mathbf{r})$. The total circulation is $\Gamma=\int \omega(\mathbf{r}) d \mathbf{r}=N \gamma$ and the average value of the energy is

$$
E=\langle H\rangle=\frac{1}{2} N(N-1) \gamma^{2} \int u\left(\left|\mathbf{r}-\mathbf{r}^{\prime}\right|\right) P_{2}\left(\mathbf{r}, \mathbf{r}^{\prime}\right) d \mathbf{r} d \mathbf{r}^{\prime}
$$

By differentiating the defining relation for $P_{j}$ and using Eq. (6), we can obtain an equilibrium hierarchy of equations for the reduced moments [18]:

$$
\frac{\partial P_{j}}{\partial \mathbf{r}_{1}}=-\frac{1}{g(E)} \frac{\partial}{\partial E}\left[g(E) P_{j}\right] \sum_{i=2}^{j} \gamma^{2} \frac{\partial u_{1, i}}{\partial \mathbf{r}_{1}}-(N-j) \gamma^{2} \int \frac{\partial u_{1, j+1}}{\partial \mathbf{r}_{1}} \frac{1}{g(E)} \frac{\partial}{\partial E}\left[g(E) P_{j+1}\right] d \mathbf{r}_{j+1} .
$$

This is the counterpart of the equilibrium hierarchy in plasma physics. It is however more complex in the present situation because it has been derived in the microcanonical ensemble. Since statistical ensembles are generically inequivalent for systems with long-range interactions, we must formulate the problem in the microcanonical ensemble which is the fundamental one. We note that the terms involving the derivative of the density of states with respect to energy can be split in two parts according to

$$
\frac{1}{g(E)} \frac{\partial}{\partial E}\left[g(E) P_{j}\right]=\beta P_{j}+\frac{\partial P_{j}}{\partial E}
$$

The terms with the $E$ derivative would not have emerged if we had started from the Gibbs canonical distribution [36]. The equivalent hierarchy of equations for material particles in interaction is given in [37]. 


\subsection{Thermodynamic limit and mean field approximation}

Since systems with long-range interactions are generically spatially inhomogeneous, the usual thermodynamic limit $N, V \rightarrow+\infty$ with $N / V$ fixed is clearly irrelevant. We define the proper thermodynamic limit of the point vortex gas as $N \rightarrow+\infty$ in such a way that the dimensionless temperature $\eta=\beta N \gamma^{2}$ and the dimensionless energy $\epsilon=E /\left(N^{2} \gamma^{2}\right)$ are fixed. It is convenient to rescale the parameters such that $\gamma \sim 1 / N, E \sim 1, \beta \sim N$ and $V \sim 1$. Then, the total circulation $\Gamma=N \gamma$ remains of order unity. We note that the ratio of $\partial P_{j} / \partial E$ on $\beta P_{j}$ is of order $1 /(E \beta)=1 /(\epsilon \eta N)$. Therefore, in the thermodynamic limit $N \rightarrow+\infty$ with $\epsilon, \eta$ fixed, the second term in Eq. (12) is always negligible with respect to the first. Using this simplification in the second equation of the equilibrium hierarchy, we get

$$
\begin{gathered}
\frac{\partial P_{1}}{\partial \mathbf{r}_{1}}\left(\mathbf{r}_{1}\right)=-\beta(N-1) \gamma^{2} \int P_{2}\left(\mathbf{r}_{1}, \mathbf{r}_{2}\right) \frac{\partial u_{12}}{\partial \mathbf{r}_{1}} d \mathbf{r}_{2}-(N-1) \gamma^{2} \int \frac{\partial u_{12}}{\partial \mathbf{r}_{1}} \frac{\partial P_{2}}{\partial E} d \mathbf{r}_{2}, \\
\frac{\partial P_{2}}{\partial \mathbf{r}_{1}}\left(\mathbf{r}_{1}, \mathbf{r}_{2}\right)=-\beta \gamma^{2} P_{2}\left(\mathbf{r}_{1}, \mathbf{r}_{2}\right) \frac{\partial u_{12}}{\partial \mathbf{r}_{1}}-\beta(N-2) \gamma^{2} \int P_{3}\left(\mathbf{r}_{1}, \mathbf{r}_{2}, \mathbf{r}_{3}\right) \frac{\partial u_{13}}{\partial \mathbf{r}_{1}} d \mathbf{r}_{3} .
\end{gathered}
$$

We now decompose the two- and three-body distribution functions in the suggestive form

$$
\begin{gathered}
P_{2}\left(\mathbf{r}_{1}, \mathbf{r}_{2}\right)=P_{1}\left(\mathbf{r}_{1}\right) P_{1}\left(\mathbf{r}_{2}\right)+P_{2}^{\prime}\left(\mathbf{r}_{1}, \mathbf{r}_{2}\right), \\
P_{3}\left(\mathbf{r}_{1}, \mathbf{r}_{2}, \mathbf{r}_{3}\right)=P_{1}\left(\mathbf{r}_{1}\right) P_{1}\left(\mathbf{r}_{2}\right) P_{1}\left(\mathbf{r}_{3}\right)+P_{2}^{\prime}\left(\mathbf{r}_{1}, \mathbf{r}_{2}\right) P_{1}\left(\mathbf{r}_{3}\right) \\
+P_{2}^{\prime}\left(\mathbf{r}_{1}, \mathbf{r}_{3}\right) P_{1}\left(\mathbf{r}_{2}\right)+P_{2}^{\prime}\left(\mathbf{r}_{2}, \mathbf{r}_{3}\right) P_{1}\left(\mathbf{r}_{1}\right)+P_{3}^{\prime}\left(\mathbf{r}_{1}, \mathbf{r}_{2}, \mathbf{r}_{3}\right) .
\end{gathered}
$$

This decomposition is the counterpart of the first terms of the Mayer expansion in plasma physics. The $P_{j}^{\prime}$ are called the cumulants or the correlation functions. Inserting these decompositions in Eqs. (13)-(14), we find after simplification that the first two equations of the equilibrium hierarchy can be written

$$
\begin{array}{r}
\frac{\partial P_{1}}{\partial \mathbf{r}_{1}}\left(\mathbf{r}_{1}\right)=-\beta(N-1) \gamma^{2} P_{1}\left(\mathbf{r}_{1}\right) \int P_{1}\left(\mathbf{r}_{2}\right) \frac{\partial u_{12}}{\partial \mathbf{r}_{1}} d \mathbf{r}_{2} \\
-\beta(N-1) \gamma^{2} \int P_{2}^{\prime}\left(\mathbf{r}_{1}, \mathbf{r}_{2}\right) \frac{\partial u_{12}}{\partial \mathbf{r}_{1}} d \mathbf{r}_{2}-(N-1) \gamma^{2} \int \frac{\partial u_{12}}{\partial \mathbf{r}_{1}} \frac{\partial P_{2}}{\partial E}\left(\mathbf{r}_{1}, \mathbf{r}_{2}\right) d \mathbf{r}_{2}, \\
\frac{\partial P_{2}^{\prime}}{\partial \mathbf{r}_{1}}\left(\mathbf{r}_{1}, \mathbf{r}_{2}\right)-(N-1) \gamma^{2} P_{1}\left(\mathbf{r}_{2}\right) \int \frac{\partial u_{13}}{\partial \mathbf{r}_{1}} \frac{\partial P_{2}}{\partial E}\left(\mathbf{r}_{1}, \mathbf{r}_{3}\right) d \mathbf{r}_{3}= \\
-\beta \gamma^{2} P_{1}\left(\mathbf{r}_{1}\right) P_{1}\left(\mathbf{r}_{2}\right) \frac{\partial u_{12}}{\partial \mathbf{r}_{1}}-\beta \gamma^{2} P_{2}^{\prime}\left(\mathbf{r}_{1}, \mathbf{r}_{2}\right) \frac{\partial u_{12}}{\partial \mathbf{r}_{1}} \\
+\beta \gamma^{2} P_{1}\left(\mathbf{r}_{1}\right) P_{1}\left(\mathbf{r}_{2}\right) \int \frac{\partial u_{13}}{\partial \mathbf{r}_{1}} P_{1}\left(\mathbf{r}_{3}\right) d \mathbf{r}_{3}-\beta(N-2) \gamma^{2} P_{2}^{\prime}\left(\mathbf{r}_{1}, \mathbf{r}_{2}\right) \int P_{1}\left(\mathbf{r}_{3}\right) \frac{\partial u_{13}}{\partial \mathbf{r}_{1}} d \mathbf{r}_{3} \\
+\beta \gamma^{2} P_{1}\left(\mathbf{r}_{2}\right) \int \frac{\partial u_{13}}{\partial \mathbf{r}_{1}} P_{2}^{\prime}\left(\mathbf{r}_{1}, \mathbf{r}_{3}\right) d \mathbf{r}_{3}-\beta(N-2) \gamma^{2} P_{1}\left(\mathbf{r}_{1}\right) \int P_{2}^{\prime}\left(\mathbf{r}_{2}, \mathbf{r}_{3}\right) \frac{\partial u_{13}}{\partial \mathbf{r}_{1}} d \mathbf{r}_{3} \\
-\beta(N-2) \gamma^{2} \int P_{3}^{\prime}\left(\mathbf{r}_{1}, \mathbf{r}_{2}, \mathbf{r}_{3}\right) \frac{\partial u_{13}}{\partial \mathbf{r}_{1}} d \mathbf{r}_{3},
\end{array}
$$

where we have used Eq. (17) to simplify some terms in Eq. (18). In the thermodynamic limit defined previously, it can be shown that the correlation functions $P_{n}^{\prime}$ are of order $N^{-(n-1)}$ [18]. Here, we shall just establish this result for the two-body distribution function $P_{2}^{\prime}$ assuming that 
it holds at higher orders. We thus neglect the term $P_{3}^{\prime}$, of order $N^{-2}$, in Eq. (18). This is the counterpart of the Kirkwood approximation in plasma physics. Then, considering the scaling of the terms in Eq. (18), we see that $P_{1} \sim 1$ and $P_{2}^{\prime} \sim \beta \gamma^{2}=\eta / N=O(1 / N)$. Therefore,

$$
P_{2}\left(\mathbf{r}_{1}, \mathbf{r}_{2}\right)=P_{1}\left(\mathbf{r}_{1}\right) P_{1}\left(\mathbf{r}_{2}\right)+O(1 / N),
$$

so that the mean field approximation $P_{2}\left(\mathbf{r}_{1}, \mathbf{r}_{2}\right) \simeq P_{1}\left(\mathbf{r}_{1}\right) P_{1}\left(\mathbf{r}_{2}\right)$ is exact for $N \rightarrow+\infty$. The coupling constant $\beta \gamma^{2} \sim 1 / N$, scaling like the inverse of the point vortex number, plays a role similar to the "plasma parameter" in plasma physics.

\subsection{The mean field equilibrium distribution}

Taking the limit $N \rightarrow+\infty$ and using Eq. (19), the first equation (17) of the equilibrium hierarchy becomes

$$
\nabla \omega(\mathbf{r})=-\beta \gamma \omega(\mathbf{r}) \nabla \int \omega\left(\mathbf{r}^{\prime}\right) u\left(\left|\mathbf{r}-\mathbf{r}^{\prime}\right|\right) d \mathbf{r}^{\prime},
$$

where $\omega(\mathbf{r})=N \gamma P_{1}(\mathbf{r})$ is the smooth vorticity field. After integration, this can be written in the form of the Boltzmann distribution

$$
\omega(\mathbf{r})=A e^{-\beta \gamma \psi(\mathbf{r})},
$$

where

$$
\psi(\mathbf{r})=\int \omega\left(\mathbf{r}^{\prime}\right) u\left(\left|\mathbf{r}-\mathbf{r}^{\prime}\right|\right) d \mathbf{r}^{\prime}
$$

is the stream function produced by the smooth distribution of point vortices. Therefore, the equilibrium density profile of the point vortices is determined by an integrodifferential equation. For the usual potential of interaction, satisfying $\Delta u=-\delta$, we find that the equilibrium vorticity profile is determined by the Boltzmann-Poisson equation

$$
-\Delta \psi=A e^{-\beta \gamma \psi(\mathbf{r})}
$$

These results can also be obtained by maximizing the Boltzmann entropy at fixed circulation and energy in order to obtain the most probable distribution of point vortices at statistical equilibrium [17]. These results can be generalized so as to take into account the conservation of the angular momentum. In that case, the stream function $\psi$ in the Boltzmann distribution is replaced by the relative stream function $\psi^{\prime}=\psi+(1 / 2) \Omega_{L} r^{2}$ [29] where $\Omega_{L}$ is a Lagrange multiplier associated with the conservation of the angular momentum (the conservation of the linear impulse can be dealt with similarly [38]).

\section{Kinetic equation from a BBGKY-like hierarchy}

\subsection{The BBGKY-like hierarchy}

We now address the out-of-equilibrium problem by using a methodology similar to the previous one. Our aim is to derive a kinetic equation for the evolution of the smooth vorticity profile $\omega(\mathbf{r}, t)$ of the point vortex gas and to see whether or not it converges to the statistical equilibrium state (21). Integrating the Liouville equation (3) on $\mathbf{r}_{j+1}, \ldots, \mathbf{r}_{N}$, it is simple to construct a hierarchy of equations for the reduced distributions. It has the form

$$
\frac{\partial P_{j}}{\partial t}+\sum_{i=1}^{j} \sum_{k=1, k \neq i}^{j} \mathbf{V}(k \rightarrow i) \frac{\partial P_{j}}{\partial \mathbf{r}_{i}}+(N-j) \sum_{i=1}^{j} \int \mathbf{V}(j+1 \rightarrow i) \frac{\partial P_{j+1}}{\partial \mathbf{r}_{i}} d \mathbf{r}_{j+1}=0 .
$$


This is the counterpart of the BBGKY hierarchy in plasma physics. The first two equations of this hierarchy are

$$
\begin{gathered}
\frac{\partial P_{1}}{\partial t}+(N-1) \frac{\partial}{\partial \mathbf{r}_{1}} \int \mathbf{V}(2 \rightarrow 1) P_{2}\left(\mathbf{r}_{1}, \mathbf{r}_{2}\right) d \mathbf{r}_{2}=0 \\
\frac{\partial P_{2}}{\partial t}+\mathbf{V}(2 \rightarrow 1) \frac{\partial P_{2}}{\partial \mathbf{r}_{1}}+(N-2) \frac{\partial}{\partial \mathbf{r}_{1}} \int \mathbf{V}(3 \rightarrow 1) P_{3}\left(\mathbf{r}_{1}, \mathbf{r}_{2}, \mathbf{r}_{3}\right) d \mathbf{r}_{3}+(1 \leftrightarrow 2)=0 .
\end{gathered}
$$

For brevity, we have not written the variable $t$ in the distribution functions. Inserting the decomposition (15) in Eq. (25), we first obtain

$$
\frac{\partial P_{1}}{\partial t}+(N-1) \frac{\partial P_{1}}{\partial \mathbf{r}_{1}} \int \mathbf{V}(2 \rightarrow 1) P_{1}\left(\mathbf{r}_{2}\right) d \mathbf{r}_{2}+(N-1) \frac{\partial}{\partial \mathbf{r}_{1}} \int \mathbf{V}(2 \rightarrow 1) P_{2}^{\prime}\left(\mathbf{r}_{1}, \mathbf{r}_{2}\right) d \mathbf{r}_{2}=0
$$

Next, substituting the decomposition (15) and (16) in Eq. (26) and using (27) to simplify some terms, we obtain

$$
\begin{array}{r}
\frac{\partial P_{2}^{\prime}}{\partial t}+\mathbf{V}(2 \rightarrow 1) \frac{\partial P_{2}^{\prime}}{\partial \mathbf{r}_{1}}+\mathbf{V}(2 \rightarrow 1) P_{1}\left(\mathbf{r}_{2}\right) \frac{\partial P_{1}}{\partial \mathbf{r}_{1}}\left(\mathbf{r}_{1}\right) \\
-P_{1}\left(\mathbf{r}_{2}\right) \frac{\partial}{\partial \mathbf{r}_{1}} \int \mathbf{V}(3 \rightarrow 1) P_{1}\left(\mathbf{r}_{1}\right) P_{1}\left(\mathbf{r}_{3}\right) d \mathbf{r}_{3} \\
-\frac{\partial}{\partial \mathbf{r}_{1}} \int \mathbf{V}(3 \rightarrow 1) P_{2}^{\prime}\left(\mathbf{r}_{1}, \mathbf{r}_{3}\right) P_{1}\left(\mathbf{r}_{2}\right) d \mathbf{r}_{3} \\
+(N-2) \frac{\partial}{\partial \mathbf{r}_{1}} \int \mathbf{V}(3 \rightarrow 1) P_{2}^{\prime}\left(\mathbf{r}_{1}, \mathbf{r}_{2}\right) P_{1}\left(\mathbf{r}_{3}\right) d \mathbf{r}_{3} \\
+(N-2) \frac{\partial}{\partial \mathbf{r}_{1}} \int \mathbf{V}(3 \rightarrow 1) P_{2}^{\prime}\left(\mathbf{r}_{2}, \mathbf{r}_{3}\right) P_{1}\left(\mathbf{r}_{1}\right) d \mathbf{r}_{3} \\
+(N-2) \frac{\partial}{\partial \mathbf{r}_{1}} \int \mathbf{V}(3 \rightarrow 1) P_{3}^{\prime}\left(\mathbf{r}_{1}, \mathbf{r}_{2}, \mathbf{r}_{3}\right) d \mathbf{r}_{3}+(1 \leftrightarrow 2)=0 .
\end{array}
$$

The equations (27) and (28) are exact for all $N$ but the hierarchy is not closed. We shall now consider the thermodynamic limit defined in Sec. 2.2. Based on the scaling of the terms in each equation of the hierarchy, we argue that there exists solutions of the whole BBGKY-like hierarchy such that the correlation functions $P_{j}^{\prime}$ scale like $1 / N^{j-1}$ at any time. This implicitly assumes that the initial condition has no correlation, or that the initial correlations respect this scaling (if there are strong initial correlations, like vortex pairs, the kinetic theory will be different from the one developed in the sequel). Recalling that $P_{1} \sim 1, P_{2}^{\prime} \sim 1 / N$ and $|\mathbf{V}(i \rightarrow j)| \sim \gamma \sim 1 / N$, we obtain at order $1 / N$ :

$$
\begin{gathered}
\frac{\partial P_{1}}{\partial t}+(N-1) \frac{\partial P_{1}}{\partial \mathbf{r}_{1}} \int \mathbf{V}(2 \rightarrow 1) P_{1}\left(\mathbf{r}_{2}\right) d \mathbf{r}_{2}+N \frac{\partial}{\partial \mathbf{r}_{1}} \int \mathbf{V}(2 \rightarrow 1) P_{2}^{\prime}\left(\mathbf{r}_{1}, \mathbf{r}_{2}\right) d \mathbf{r}_{2}=0 \\
\frac{\partial P_{2}^{\prime}}{\partial t}+\left[\mathbf{V}(2 \rightarrow 1)-\int \mathbf{V}(3 \rightarrow 1) P_{1}\left(\mathbf{r}_{3}\right) d \mathbf{r}_{3}\right] P_{1}\left(\mathbf{r}_{2}\right) \frac{\partial P_{1}}{\partial \mathbf{r}_{1}}\left(\mathbf{r}_{1}\right) \\
+N \frac{\partial P_{2}^{\prime}}{\partial \mathbf{r}_{1}} \int \mathbf{V}(3 \rightarrow 1) P_{1}\left(\mathbf{r}_{3}\right) d \mathbf{r}_{3}+N \frac{\partial}{\partial \mathbf{r}_{1}} \int \mathbf{V}(3 \rightarrow 1) P_{2}^{\prime}\left(\mathbf{r}_{2}, \mathbf{r}_{3}\right) P_{1}\left(\mathbf{r}_{1}\right) d \mathbf{r}_{3}+(1 \leftrightarrow 2)=0
\end{gathered}
$$

The three-body correlation function can be neglected. If we introduce the notations $\omega=N \gamma P_{1}$ (smooth vorticity field) and $g=N^{2} P_{2}^{\prime}$ (two-body correlation function), these equations can be rewritten

$$
\frac{\partial \omega_{1}}{\partial t}+\frac{N-1}{N}\langle\mathbf{V}\rangle_{1} \frac{\partial \omega_{1}}{\partial \mathbf{r}_{1}}=-\gamma \frac{\partial}{\partial \mathbf{r}_{1}} \int \mathbf{V}(2 \rightarrow 1) g\left(\mathbf{r}_{1}, \mathbf{r}_{2}\right) d \mathbf{r}_{2}
$$




$$
\begin{array}{r}
\frac{\partial g}{\partial t}+\langle\mathbf{V}\rangle_{1} \frac{\partial g}{\partial \mathbf{r}_{1}}+\frac{1}{\gamma^{2}} \mathcal{V}(2 \rightarrow 1) \omega_{2} \frac{\partial \omega_{1}}{\partial \mathbf{r}_{1}} \\
+\frac{\partial}{\partial \mathbf{r}_{1}} \int \mathbf{V}(3 \rightarrow 1) g\left(\mathbf{r}_{2}, \mathbf{r}_{3}, t\right) \frac{\omega_{1}}{\gamma} d \mathbf{r}_{3}+(1 \leftrightarrow 2)=0 .
\end{array}
$$

For brevity, we have used the abbreviations $\omega_{1}=\omega\left(\mathbf{r}_{1}, t\right)$ and $\omega_{2}=\omega\left(\mathbf{r}_{2}, t\right)$. We have also introduced the mean velocity in $\mathbf{r}_{1}$ created by all the vortices

$$
\langle\mathbf{V}\rangle_{1}=\int \mathbf{V}(2 \rightarrow 1) \frac{\omega_{2}}{\gamma} d \mathbf{r}_{2},
$$

and the fluctuating velocity created by point vortex 2 on point vortex 1 :

$$
\mathcal{V}(2 \rightarrow 1)=\mathbf{V}(2 \rightarrow 1)-\frac{1}{N}\langle\mathbf{V}\rangle_{1} .
$$

These equations (31)-(32) are exact at order $O(1 / N)$. They form therefore the right basis to develop a kinetic theory.

(i) Collisionless regime: If we consider the limit $N \rightarrow+\infty$ (for a fixed time $t$ ), noting that $P_{2}^{\prime}=O(1 / N) \rightarrow 0$, we find that the smooth vorticity field $\omega(\mathbf{r}, t)$ of the point vortex gas is solution of the 2D Euler equation

$$
\frac{\partial \omega}{\partial t}+\langle\mathbf{V}\rangle \nabla \omega=0, \quad\langle\mathbf{V}\rangle=-\mathbf{z} \times \nabla \psi,
$$

where the stream function $\psi(\mathbf{r}, t)$ is given by Eq. (22) with $\omega(\mathbf{r}, t)$ instead of $\omega(\mathbf{r})$. The 2D Euler equation is valid when the correlations between point vortices can be neglected, i.e. $P_{2}\left(\mathbf{r}_{1}, \mathbf{r}_{2}, t\right)=P_{1}\left(\mathbf{r}_{1}, t\right) P_{2}\left(\mathbf{r}_{2}, t\right)$, which is the case for $N \rightarrow+\infty$. The Euler equation describes the collisionless evolution of the point vortex gas up to a time of order $N t_{D}$ (where $t_{D}$ is the dynamical time) at least. In practice, $N \geq 1000$ so that the domain of validity of the $2 \mathrm{D}$ Euler equation is huge. The Euler equation is the counterpart of the Vlasov equation in plasma physics and stellar dynamics. It can undergo a process of violent relaxation towards a Quasi Stationary State 31, 32, 33, 34, as discussed in Secs. 4.2 and 4.3,

(ii) Collisional regime: If we want to describe the collisional evolution of the point vortex gas, we need to consider finite $N$ effects. Equations (31)-(32) describe the evolution of the system on a timescale of order $N t_{D}$. The equation for the evolution of the smooth vorticity field is of the form

$$
\frac{\partial \omega}{\partial t}+\frac{N-1}{N}\langle\mathbf{V}\rangle \nabla \omega=C_{N}[\omega]
$$

where $C_{N}$ is a "collision" term analogous to the one arising in the Boltzmann equation. In the present context, there are not real collisions between point vortices. The term on the right hand side of Eq. (36) is due to the development of correlations between vortices as time goes on. It is related to the two-body correlation function $g\left(\mathbf{r}_{1}, \mathbf{r}_{2}, t\right)$ which is determined in terms of the vorticity by Eq. (32). Our aim is to obtain an expression for the collision term $C_{N}[\omega]$ at the order $1 / N$. The difficulty with Eq. (32) for the two-body correlation function is that it is an integrodifferential equation. The second term is an advective term, the third term is the source of the correlation and the fourth term takes into account collective effects. In this paper, we shall neglect the contribution of the integral in Eq. (32). Then, we get the coupled system

$$
\frac{\partial \omega_{1}}{\partial t}+\frac{N-1}{N}\langle\mathbf{V}\rangle_{1} \frac{\partial \omega_{1}}{\partial \mathbf{r}_{1}}=-\gamma \frac{\partial}{\partial \mathbf{r}_{1}} \int \mathbf{V}(2 \rightarrow 1) g\left(\mathbf{r}_{1}, \mathbf{r}_{2}\right) d \mathbf{r}_{2}
$$




$$
\frac{\partial g}{\partial t}+\left[\langle\mathbf{V}\rangle_{1} \frac{\partial}{\partial \mathbf{r}_{1}}+\langle\mathbf{V}\rangle_{2} \frac{\partial}{\partial \mathbf{r}_{2}}\right] g+\left[\mathcal{V}(2 \rightarrow 1) \frac{\partial}{\partial \mathbf{r}_{1}}+\mathcal{V}(1 \rightarrow 2) \frac{\partial}{\partial \mathbf{r}_{2}}\right] \frac{\omega_{1}}{\gamma} \frac{\omega_{2}}{\gamma}=0
$$

The integral that we have neglected contains "collective effects" that are taken into account in the approach of Dubin \& O'Neil [20]. However, their study is restricted to axisymmetric flows and makes a Markovian approximation. These assumptions are necessary to use LaplaceFourier transforms in order to solve the integro-differential equation (32). Alternatively, if we neglect collective effects, we can obtain a general kinetic equation in a closed form (52) that is valid for flows that are not necessarily axisymmetric and that can take into account memory effects. This equation has interest in its own right (despite its limitations) because its structure bears a lot of physical significance. Before deriving this general equation, we shall first consider the case of axisymmetric flows and obtain a simple explicit kinetic equation valid for such flows when collective effects are neglected.

\subsection{The Markovian axisymmetric equation}

For an axisymmetric flow, the vorticity field and the two-point correlation function can be written as $\omega=\omega(r, t)$ and $g=g\left(r_{1}, r_{2}, \theta_{1}-\theta_{2}, t\right)$, and the mean velocity as $\langle\mathbf{V}\rangle=\langle V\rangle_{\theta}(r, t) \mathbf{e}_{\theta}$. On the other hand, the projection of $\mathbf{V}(2 \rightarrow 1)$ in the direction of $\mathbf{r}_{1}$ is

$$
V_{r_{1}}(2 \rightarrow 1)=\gamma \frac{1}{r_{1}} \frac{\partial u_{12}}{\partial \theta_{1}}
$$

where $u_{12}=u\left(r_{1}, r_{2}, \theta_{1}-\theta_{2}\right)$ is symmetric in $r_{1}$ and $r_{2}$ (see Appendix $\underline{\mathrm{A}}$ ). In that case, Eqs. (37)-(38) become

$$
\begin{gathered}
\frac{\partial \omega_{1}}{\partial t}=-\gamma^{2} \frac{1}{r_{1}} \frac{\partial}{\partial r_{1}} \int_{0}^{+\infty} r_{2} d r_{2} \int_{0}^{2 \pi} \frac{\partial u}{\partial \phi} g\left(r_{1}, r_{2}, \phi, t\right) d \phi \\
\frac{\partial g}{\partial t}+\left[\Omega\left(r_{1}, t\right)-\Omega\left(r_{2}, t\right)\right] \frac{\partial g}{\partial \phi}=-\frac{\partial u}{\partial \phi}\left(\frac{1}{r_{1}} \frac{\partial}{\partial r_{1}}-\frac{1}{r_{2}} \frac{\partial}{\partial r_{2}}\right) \omega\left(r_{1}, t\right) \frac{\omega}{\gamma}\left(r_{2}, t\right),
\end{gathered}
$$

where we have set $\phi=\theta_{1}-\theta_{2}$ and where $\Omega(r, t)=\langle V\rangle_{\theta}(r, t) / r$ is the angular velocity of the mean flow. Taking the Fourier transform of Eq. (41) with respect to $\phi$ and introducing the notations $\partial=\left(1 / r_{1}\right) \partial / \partial r_{1}-\left(1 / r_{2}\right) \partial / \partial r_{2}, \omega_{1}=\omega\left(r_{1}, t\right), \omega_{2}=\omega\left(r_{2}, t\right)$ and $\Delta \Omega=\Omega\left(r_{1}, t\right)-\Omega\left(r_{2}, t\right)$, we obtain

$$
\frac{d \hat{g}_{m}}{d t}+i m \Delta \Omega \hat{g}_{m}=-\frac{i}{\gamma} m \hat{u}_{m} \partial \omega_{1} \omega_{2} .
$$

The Fourier transform of the potential of interaction $u$ is discussed in Appendix A where explicit examples are considered. In terms of the Fourier transform of the correlation function, the kinetic equation (40) can be rewritten

$$
\frac{\partial \omega_{1}}{\partial t}=-2 \pi \gamma^{2} \frac{1}{r_{1}} \frac{\partial}{\partial r_{1}} \int_{0}^{+\infty} r_{2} d r_{2} \sum_{m} m \hat{u}_{m} \operatorname{Im} \hat{g}_{m}\left(r_{1}, r_{2}, t\right)
$$

We shall assume that $\operatorname{Im} \hat{g}_{m}\left(r_{1}, r_{2}, t\right)$ relaxes on a timescale which is much smaller than the timescale on which $\omega(r, t)$ changes. This is the equivalent of the Bogoliubov hypothesis in plasma physics. If we ignore memory effects, we can integrate the first order differential equation (42) by considering the last term as a constant. This yields

$$
\hat{g}_{m}\left(r_{1}, r_{2}, t\right)=-\int_{0}^{t} d \tau \frac{i}{\gamma} m \hat{u}_{m} e^{-i m \Delta \Omega \tau} \partial \omega_{1} \omega_{2}
$$


where we have assumed that no correlation is present initially: $g(t=0)=0$. Then, we can replace $\operatorname{Im} \hat{g}_{m}\left(r_{1}, r_{2}, t\right)$ in Eq. (43) by its value obtained for $t \rightarrow+\infty$, which reads

$$
\operatorname{Im} \hat{g}_{m}\left(r_{1}, r_{2},+\infty\right)=-\frac{\pi}{\gamma} m \hat{u}_{m} \delta(m \Delta \Omega) \partial \omega_{1} \omega_{2}
$$

Substituting this relation in Eq. (43), we obtain the kinetic equation

$$
\frac{\partial \omega_{1}}{\partial t}=2 \pi^{2} \gamma \frac{1}{r_{1}} \frac{\partial}{\partial r_{1}} \int_{0}^{+\infty} r_{2} d r_{2} \chi\left(r_{1}, r_{2}\right) \delta\left(\Omega_{1}-\Omega_{2}\right)\left(\frac{1}{r_{1}} \omega_{2} \frac{\partial \omega_{1}}{\partial r_{1}}-\frac{1}{r_{2}} \omega_{1} \frac{\partial \omega_{2}}{\partial r_{2}}\right)
$$

where we have defined

$$
\chi\left(r_{1}, r_{2}\right)=\sum_{m}|m| \hat{u}_{m}\left(r_{1}, r_{2}\right)^{2}
$$

For the potential of interaction (158), this function is given by Eq. (161) and we recover the kinetic equation obtained in [23]:

$$
\frac{\partial \omega_{1}}{\partial t}=-\frac{\gamma}{4} \frac{1}{r_{1}} \frac{\partial}{\partial r_{1}} \int_{0}^{+\infty} r_{2} d r_{2} \ln \left[1-\left(\frac{r_{<}}{r_{>}}\right)^{2}\right] \delta\left(\Omega_{1}-\Omega_{2}\right)\left(\frac{1}{r_{1}} \omega_{2} \frac{\partial \omega_{1}}{\partial r_{1}}-\frac{1}{r_{2}} \omega_{1} \frac{\partial \omega_{2}}{\partial r_{2}}\right) .
$$

This equation, which ignores collective effects, is the vortex analogue of the Landau equation in plasma physics. We can show [23, 29] that it conserves $\Gamma, E, L$, that it satisfies an $H$ theorem $(\dot{S} \geq 0)$ and that the Boltzmann distribution (60) is a particular steady state, but not the only one (see [29] for more discussion). Collective effects can be taken into account by keeping the contribution of the last integral in Eq. (32). For axisymmetric flows, these terms could be evaluated at the price of complicated calculations in the complex plane similar to those performed in plasma physics to derived the Lenard-Balescu equation from the BBGKY hierarchy. It would be interesting to make this derivation although it will not be attempted in the present paper. This would certainly lead to the kinetic equation derived by Dubin \& O'Neil [20] from a quasilinear theory of the Klimontovich equation. As we shall see, the consideration of collective effects is equivalent to replacing the bare potential of interaction by an "effective potential". The resulting kinetic equation remains of the form of Eq. (46) with a modified function $\chi_{P}\left(r_{1}, r_{2}\right)$. Therefore, as far as the general structure of the kinetic equations is concerned, our simple treatment is of interest. Furthermore, it can be generalized to non axisymmetric flows as considered in the next section. Finally, since the diffusion coefficient in Eq. (46) does not diverge (contrary to the 3D Landau equation in plasma physics), the Lenard-Balescu treatment of collective effects is not necessary in our case for a first analysis.

\subsection{The general non Markovian kinetic equation}

The above kinetic equations assume that the flow is axisymmetric and rely on the assumption that the correlation function relaxes much more rapidly than the vorticity field. The Markovian approximation is expected to be a good approximation in the limit $N \rightarrow+\infty$ that we consider since the vorticity profile changes only on a timescale of order $N t_{D}$ (where $t_{D}$ is the dynamical time) or even larger. However, for systems with long-range interactions, there can be situations where the decorrelation time of the fluctuations is very long so that the Markovian approximation may not be completely justified (this is the case for self-gravitating systems). For comparison, and for sake of generality, it can be of interest to derive non-markovian kinetic 
equations for point vortices. For an arbitrary flow, Eq. (38) for the correlation function can be written

$$
\frac{\partial g}{\partial t}+\mathcal{L} g=-\left[\mathcal{V}(2 \rightarrow 1) \frac{\partial}{\partial \mathbf{r}_{1}}+\mathcal{V}(1 \rightarrow 2) \frac{\partial}{\partial \mathbf{r}_{2}}\right] \frac{\omega}{\gamma}\left(\mathbf{r}_{1}, t\right) \frac{\omega}{\gamma}\left(\mathbf{r}_{2}, t\right)
$$

where we have denoted the advective term by $\mathcal{L}$ (Liouvillian operator). Solving formally this equation with the Green function

$$
G\left(t, t^{\prime}\right)=\exp \left\{-\int_{t^{\prime}}^{t} \mathcal{L}(\tau) d \tau\right\}
$$

we obtain

$$
g\left(\mathbf{r}_{1}, \mathbf{r}_{2}, t\right)=-\int_{0}^{t} d \tau G(t, t-\tau)\left[\mathcal{V}(2 \rightarrow 1) \frac{\partial}{\partial \mathbf{r}_{1}}+\mathcal{V}(1 \rightarrow 2) \frac{\partial}{\partial \mathbf{r}_{2}}\right] \frac{\omega}{\gamma}\left(\mathbf{r}_{1}, t-\tau\right) \frac{\omega}{\gamma}\left(\mathbf{r}_{2}, t-\tau\right)
$$

The Green function constructed with the smooth velocity field $\langle\mathbf{V}\rangle$ means that, in order to evaluate the time integral in Eq. (51), we must move the coordinates $\mathbf{r}_{i}(t-\tau)$ of the point vortices with the mean field flow $\langle\mathbf{V}\rangle(\mathbf{r}, t)$, adopting a Lagrangian point of view. Thus, in evaluating the integral, the coordinates $\mathbf{r}_{i}$ must be viewed as $\mathbf{r}_{i}(t-\tau)$, where $\mathbf{r}_{i}(t-\tau)=$ $\mathbf{r}_{i}(t)-\int_{0}^{\tau} d s\langle\mathbf{V}\rangle\left(\mathbf{r}_{i}(t-s), t-s\right) d s$. Substituting this result in Eq. (37), we obtain

$$
\begin{aligned}
\frac{\partial \omega_{1}}{\partial t} & +\frac{N-1}{N}\langle\mathbf{V}\rangle_{1} \frac{\partial \omega}{\partial \mathbf{r}_{1}}=\frac{\partial}{\partial r_{1}^{\mu}} \int_{0}^{t} d \tau \int d \mathbf{r}_{2} V^{\mu}(2 \rightarrow 1, t) G(t, t-\tau) \\
& \times\left[\mathcal{V}^{\nu}(2 \rightarrow 1) \frac{\partial}{\partial r_{1}^{\nu}}+\mathcal{V}^{\nu}(1 \rightarrow 2) \frac{\partial}{\partial r_{2}^{\nu}}\right] \omega\left(\mathbf{r}_{1}, t-\tau\right) \frac{\omega}{\gamma}\left(\mathbf{r}_{2}, t-\tau\right)
\end{aligned}
$$

This returns the general kinetic equation obtained by Chavanis [23] with the projection operator formalism (note that we can replace $V^{\mu}(2 \rightarrow 1, t)$ by $\mathcal{V}^{\mu}(2 \rightarrow 1, t)$ in the first term of the r.h.s. of the equation since the fluctuations vanish in average). It slightly differs from the equation obtained in [23] by a term $(N-1) / N$ in the 1.h.s. This new derivation of the kinetic equation (52) from a systematic expansion of the solutions of the BBGKY hierarchy in powers of $1 / N$ is valuable because the formalism is much simpler than the projection operator formalism and clearly shows which terms have been neglected in the derivation. It also clearly shows that the kinetic equation (52) is valid at order $1 / N$ so that it describes the system on a timescale of order $N t_{D}$. In [8, 29], we had obtained this estimate a posteriori.

\subsection{Summary of the different kinetic equations}

Let us briefly summarize the different kinetic equations that appeared in our analysis. When collective effects are ignored, the kinetic equation describing the evolution of the system as a whole at order $1 / N$ is

$$
\begin{aligned}
& \frac{\partial \omega}{\partial t}+\frac{N-1}{N}\langle\mathbf{V}\rangle \nabla \omega=\frac{\partial}{\partial r^{\mu}} \int_{0}^{t} d \tau \int d \mathbf{r}_{1} V^{\mu}(1 \rightarrow 0) G(t, t-\tau) \\
& \quad \times\left\{\mathcal{V}^{\nu}(1 \rightarrow 0) \frac{\partial}{\partial r^{\nu}}+\mathcal{V}^{\nu}(0 \rightarrow 1) \frac{\partial}{\partial r_{1}^{\nu}}\right\} \omega(\mathbf{r}, t-\tau) \frac{\omega}{\gamma}\left(\mathbf{r}_{1}, t-\tau\right) .
\end{aligned}
$$


If we make a Markov approximation and extend the time integral to infinity, we obtain

$$
\begin{aligned}
\frac{\partial \omega}{\partial t}+\frac{N-1}{N} & \langle\mathbf{V}\rangle \nabla \omega=\frac{\partial}{\partial r^{\mu}} \int_{0}^{+\infty} d \tau \int d \mathbf{r}_{1} V^{\mu}(1 \rightarrow 0) G(t, t-\tau) \\
\times & \left\{\mathcal{V}^{\nu}(1 \rightarrow 0) \frac{\partial}{\partial r^{\nu}}+\mathcal{V}^{\nu}(0 \rightarrow 1) \frac{\partial}{\partial r_{1}^{\nu}}\right\} \omega(\mathbf{r}, t) \frac{\omega}{\gamma}\left(\mathbf{r}_{1}, t\right) .
\end{aligned}
$$

As we have indicated, the Markov approximation is justified for $N \rightarrow+\infty$ because the timescale $N t_{D}$ on which $\omega$ changes is long compared to the timescale $\tau_{\text {corr }}$ for which the integrand in Eq. (54) has significant support. We do not assume that the decorrelation time is extremely short so that, in the time integral, the vorticity and the vorticity gradient must be evaluated at $\mathbf{r}(t-\tau)$ and $\mathbf{r}_{1}(t-\tau)$ where now $\mathbf{r}_{i}(t-\tau)=\mathbf{r}_{i}(t)-\int_{0}^{\tau} d s\langle\mathbf{V}\rangle\left(\mathbf{r}_{i}(t-s), t\right) d s$. On the other hand, for an axisymmetric evolution, using the relation (153) and $r_{i}(t-\tau)=r_{i}(t)$ and $\theta_{i}(t-\tau)=\theta_{i}(t)-\Omega\left(r_{i}(t), t\right) \tau$, Eq. (53) takes the form

$$
\begin{array}{r}
\frac{\partial \omega}{\partial t}=\frac{1}{r} \frac{\partial}{\partial r} r \int_{0}^{t} d \tau \int_{0}^{2 \pi} d \theta_{1} \int_{0}^{+\infty} r r_{1} d r_{1} V_{r}(1 \rightarrow 0, t) \\
\times V_{r}(1 \rightarrow 0, t-\tau)\left(\frac{1}{r} \frac{\partial}{\partial r}-\frac{1}{r_{1}} \frac{\partial}{\partial r_{1}}\right) \omega(r, t-\tau) \frac{\omega}{\gamma}\left(r_{1}, t-\tau\right) .
\end{array}
$$

The integral on $\theta_{1}$ can be performed using Eq. (98), and we get

$$
\begin{array}{rl}
\frac{\partial \omega}{\partial t}=2 \pi \gamma \frac{1}{r} \frac{\partial}{\partial r} \int_{0}^{t} & d \tau \int_{0}^{+\infty} r_{1} d r_{1} \sum_{m} m^{2} \hat{u}_{m}^{2}\left(r, r_{1}\right) \cos (m \Delta \Omega \tau) \\
& \times\left(\frac{1}{r} \frac{\partial}{\partial r}-\frac{1}{r_{1}} \frac{\partial}{\partial r_{1}}\right) \omega(r, t-\tau) \omega\left(r_{1}, t-\tau\right)
\end{array}
$$

This equation can also be obtained from the approach of Sec. 3.2 by keeping memory effects in Eq. (44). If we make a Markovian approximation $\omega\left(r_{1}, t-\tau\right) \simeq \omega\left(r_{1}, t\right)$ and $\omega(r, t-\tau) \simeq \omega(r, t)$, and extend the time integration to $+\infty$ in Eq. (55), we obtain

$$
\begin{aligned}
\frac{\partial \omega}{\partial t} & =\frac{1}{r} \frac{\partial}{\partial r} r \int_{0}^{+\infty} d \tau \int_{0}^{2 \pi} d \theta_{1} \int_{0}^{+\infty} r r_{1} d r_{1} V_{r}(1 \rightarrow 0, t) \\
& \times V_{r}(1 \rightarrow 0, t-\tau)\left(\frac{1}{r} \frac{\partial}{\partial r}-\frac{1}{r_{1}} \frac{\partial}{\partial r_{1}}\right) \omega(r, t) \frac{\omega}{\gamma}\left(r_{1}, t\right)
\end{aligned}
$$

The integral on $\tau$ and $\theta_{1}$ can be performed using Eq. (98), see [23, 29] for details, and we get the kinetic equation (46). If we make the approximation $\omega\left(r_{1}, t-\tau\right) \simeq \omega\left(r_{1}, t\right)$ and $\omega(r, t-$ $\tau) \simeq \omega(r, t)$ but keep the time integration from 0 to $t$, we obtain the equation derived in [29] incorporating a function $M(t)$ which regularizes the delta function occuring in Eq. (46). Finally, in Appendix B, we propose a simple heuristic kinetic equation that may be of interest.

\subsection{Discussion}

These kinetic equations possess a lot of interesting properties. Let us first consider the Markovian axisymmetric equation (46). The collisional evolution of point vortices is truly due to long range interactions because the current in $r$ is caused by "distant collisions" with vortices located in $r_{1} \neq r$ that can be far away. This is different from plasma physics and stellar dynamics where the collisions are assumed to be local [39]. Therefore, the current occurs only in velocity space and is due to "collisions" involving particles at the same location but having different 
velocities $v_{1} \neq v$ (recall that the position $r$ in the point vortex system plays the same role as the velocity $v$ in the plasma system). Therefore, in the case of stellar systems and plasmas, the collisional term is determined as if the system were spatially homogeneous. For these systems, long-range interactions manifest themselves only as mean field effects in the advective term (Vlasov) of the kinetic equation (see Eq. (44) of [40]). By contrast, the point vortex gas is the first system where collisions involve distant particles. The collisional evolution in $r$ is due to a condition of resonance $\Omega\left(r_{1}, t\right)=\Omega(r, t)$ with point vortices in $r_{1} \neq r$ that have the same angular velocity. Clearly, this condition can be satisfied only when the profile of angular velocity is non-monotonic [20, 23]. Therefore, the evolution stops when the profile of angular velocity becomes monotonic (so that there is no resonance) even if the system has not reached statistical equilibrium. In that case, the system settles on a Quasi Stationary State (QSS) that is not the Boltzmann distribution (21) predicted by statistical mechanics [29]. On the timescale $N t_{D}$ on which the kinetic theory is valid, the collisions tend to create a monotonic profile of angular velocity. Since the entropy increases monotonically, the vorticity profile tends to approach the Boltzmann distribution but does not attain it in general because of the absence of resonances. The Boltzmann distribution may be reached on longer timescales, larger than $N t_{D}$. To describe this regime, we need to determine terms of order $N^{-2}$ or smaller in the expansion of the solutions of the BBGKY hierarchy for $N \rightarrow+\infty$. This implies in particular the determination of the three-body correlation function, which is a formidable task. It is interesting to note that the markovian axisymmetric kinetic equation (46) conserves all the integral constraints of the point vortex dynamics (circulation, energy, angular momentum) and satisfies an H-theorem for the Boltzmann entropy, so that the entropy is non-decreasing $\dot{S} \geq 0$ [29]. However, as we have indicated previously, this kinetic equation does not in general converge towards the Boltzmann distribution. This is because this kinetic equation admits an infinite number of stationary solutions among which the Boltzmann distribution is just a particular case (see [29] for a detailed discussion). This is at variance with the Landau and Lenard-Balescu equations which always converge towards the Boltzmann distribution [40]. In these equations, the collisional evolution of the system is also due to a condition of resonance $\mathbf{k} \cdot \mathbf{v}_{1}=\mathbf{k} \cdot \mathbf{v}$ (see Eq. (49) of [40]) but the Boltzmann distribution is the only steady state of these kinetic equations. As noted in [29], the kinetic theory of point vortices is more closely related to the kinetic theory of one-dimensional systems with long-range interactions (like the HMF model) for which the collision term vanishes identically at order $1 / N$ [41, 42, 40].

Let us now consider the more general kinetic equation (52). We can prove by a direct calculation that this equation conserves the angular momentum and the linear impulse (see Appendix D of [23]). The conservation of the energy is more difficult to establish by a direct calculation but since Eq. (52) is exact at order $O(1 / N)$, the energy must be conserved (the integral constraints must be conserved at any order). Finally, we note that we cannot prove the $H$-theorem. It is only when additional approximations are made (markovian approximation) that the $H$-theorem is obtained (see Sec. 3.2 and [29]). To be more precise, let us compute the rate of change of the Boltzmann entropy for point vortices $S=-\int \frac{\omega}{\gamma} \ln \frac{\omega}{\gamma} d \mathbf{r}$ with respect to the general kinetic equation (52). After straightforward manipulations, it can be put in the form

$$
\begin{array}{rl}
\dot{S}=\frac{1}{2 \gamma^{2}} \int d & \mathbf{r} d \mathbf{r}_{1} \frac{1}{\omega \omega_{1}} \int_{0}^{t} d \tau\left[\mathcal{V}^{\mu}(1 \rightarrow 0) \omega_{1} \frac{\partial \omega}{\partial r^{\mu}}+\mathcal{V}^{\mu}(0 \rightarrow 1) \omega \frac{\partial \omega_{1}}{\partial r_{1}^{\mu}}\right]_{t} \\
\times G(t, t-\tau)\left[\mathcal{V}^{\nu}(1 \rightarrow 0) \omega_{1} \frac{\partial \omega}{\partial r^{\nu}}+\mathcal{V}^{\nu}(0 \rightarrow 1) \omega \frac{\partial \omega_{1}}{\partial r_{1}^{\nu}}\right]_{t-\tau} .
\end{array}
$$

We note that, because of memory terms, the monotonic increase of the entropy is not granted. In the case of point vortices, the decorrelation time is much shorter than the relaxation time 
(of order $N t_{D}$ or larger) so that the markovian approximation is justified for $N \rightarrow+\infty$. In that case the entropy monotonically increases as shown explicitly for axisymmetric flows (see Sec. 3.2 and [29]). However, as we have already indicated for the axisymmetric markovian equation (46), even if $\dot{S} \geq 0$ and $\dot{E}=\dot{\Gamma}=0$, this does not imply that the kinetic equation will relax towards the Boltzmann distribution of statistical equilibrium [29. Indeed, the relaxation can stop before in the absence of resonances. The same remark applies to the more general equation (52), valid for non-axisymmetric flows, although this is more difficult to see. In order to make it clearer, one possibility would be to use the timescale separation between the dynamical time $t_{D}$ and the collisional time $t_{\text {coll }} \sim N t_{D}$ and derive an "orbit-averaged" kinetic equation in terms of appropriate variables similar to the angle-action variables used in other contexts. In that case, we would get a simpler kinetic equation, similar to the one derived in [43, and exhibiting an appropriate form of "resonances" between different orbits. This would generalize the condition of resonance $\Omega(r)=\Omega\left(r^{\prime}\right)$ associated to Eq. (46) to the case of non-axisymmetric flows. Note that a phenomenological equation, valid for general flows, and exhibiting a form of "resonances" required to ensure the conservation of the energy is provided by Eq. (137) of [23] (see also Appendix B).

\subsection{The growth of correlations}

In Sec. 3.2, we have derived the equation satisfied by the two-body correlation function $g\left(r_{1}, r_{2}, \phi, t\right)$ at order $1 / N$ for an axisymmetric evolution. To derive the kinetic equation (46)), we only had to determine the imaginary part of the Fourier transform of $g$ for $t \rightarrow+\infty$. In this section, we discuss the growth of the two-body correlation function in more detail. It is determined by the equation (41), i.e.:

$$
\frac{\partial g}{\partial t}+\Delta \Omega \frac{\partial g}{\partial \phi}=-\frac{1}{\gamma} \frac{\partial u}{\partial \phi} \partial \omega_{1} \omega_{2} .
$$

We shall assume that the initial vorticity profile is the Boltzmann distribution of statistical equilibrium

$$
\omega=A e^{-\beta \gamma\left(\psi+\frac{1}{2} \Omega_{L} r^{2}\right)} .
$$

This distribution is a stationary solution of Eq. (46). Therefore, the r.h.s. of Eq. (59) is independent on time. Using Eq. (60) and the relations

$$
\langle V\rangle_{\theta}(r, t)=-\frac{\partial \psi}{\partial r}(r, t)=\Omega(r, t) r,
$$

we find that

$$
\partial \omega_{1} \omega_{2}=\beta \gamma \omega_{1} \omega_{2} \Delta \Omega .
$$

Substituting this result in Eq. (59) and introducing the function $h=h\left(\phi, r_{1}, r_{2}, t\right)$ through the relation $g=\omega_{1} \omega_{2} h$, we get

$$
\frac{\partial h}{\partial t}+\Delta \Omega \frac{\partial h}{\partial \phi}=-\beta \Delta \Omega \frac{\partial u}{\partial \phi} .
$$

Taking the Fourier transform of the foregoing equation and integrating on time, we obtain

$$
\hat{h}_{m}(t)=-i \beta \int_{0}^{t} d \tau m \Delta \Omega \hat{u}_{m} e^{-i m \Delta \Omega \tau}=\beta \hat{u}_{m}\left(e^{-i m \Delta \Omega t}-1\right) .
$$


Therefore

$$
\hat{g}_{m}\left(r_{1}, r_{2}, t\right)=\beta \hat{u}_{m}\left(e^{-i m \Delta \Omega t}-1\right) \omega_{1} \omega_{2}
$$

We note that $\operatorname{Im}\left(\hat{g}_{m}\right)$ has a limit (45) for $t \rightarrow+\infty$, while $\operatorname{Re}\left(\hat{g}_{m}\right)$ has no limit. Taking the inverse Fourier transform of Eq. (65), we obtain

$$
g\left(\phi, r_{1}, r_{2}, t\right)=\beta\left[u\left(r_{1}, r_{2}, \phi-\Delta \Omega t\right)-u\left(r_{1}, r_{2}, \phi\right)\right] \omega_{1} \omega_{2}
$$

This equation describes the growth of two-body correlations in an axisymmetric flow assuming that the one-body distribution is given by the Boltzmann distribution. For the potential of interaction (158) written in the form

$$
u_{12}=-\frac{1}{4 \pi} \ln \left(r_{1}^{2}+r_{2}^{2}-2 r_{1} r_{2} \cos \phi\right)
$$

the correlation function (66) is explicitly given by

$$
g\left(\phi, r_{1}, r_{2}, t\right)=-\frac{\beta}{4 \pi} \ln \left[\frac{r_{1}^{2}+r_{2}^{2}-2 r_{1} r_{2} \cos (\phi-\Delta \Omega t)}{r_{1}^{2}+r_{2}^{2}-2 r_{1} r_{2} \cos \phi}\right] \omega_{1} \omega_{2} .
$$

We note that $g$ has an oscillatory behavior, so that it has no limit for $t \rightarrow+\infty$. We recall that our approach is valid at order $1 / N$. Therefore, it corresponds to a linear regime extending on a timescale of order $N t_{D}$. The two-body correlation function may reach the value it has at statistical equilibrium on a longer timescale but next order terms in $1 / N$ in the developement must be taken into account.

\section{Kinetic equations from a quasilinear theory}

In this section, we show that the general kinetic equation (52) describing the collisional evolution of the point vortex gas can also be derived from a quasilinear theory of the Klimontovich equation. We will compare the results with the quasilinear theory of the 2D Euler equation developed in [30] to describe the process of violent relaxation in the collisionless regime.

\subsection{The slow collisional evolution of point vortices}

The exact vorticity profile of a gas of point vortices is a sum of Dirac functions given by Eq. (1). It satisfies the equation

$$
\frac{\partial \omega_{d}}{\partial t}+\mathbf{u}_{d} \nabla \omega_{d}=0
$$

where $\mathbf{u}_{d}=-\mathbf{z} \times \nabla \psi_{d}$ is the exact velocity field created by $\omega_{d}$. Equation (69) is the counterpart of the Klimontovich equation in plasma physics. It should not be confused with the 2D Euler equation (35) or (81), the counterpart of the Vlasov equation, which has the same mathematical structure but which applies to the smooth vorticity field $\omega$. The $2 \mathrm{D}$ Euler equation is valid during the collisionless regime (see next section) while the Klimontovich equation is exact and strictly contains the same information as the Hamiltonian equations (2). We now decompose the exact vorticity field in the form $\omega_{d}=\omega+\delta \omega$ where $\omega=\left\langle\omega_{d}\right\rangle$ is the smooth vorticity and $\delta \omega$ the fluctuation around it. Substituting this decomposition in Eq. (69) and locally averaging over the fluctuations, we get

$$
\frac{\partial \omega}{\partial t}+L \omega=-\langle\delta \mathbf{u} \nabla \delta \omega\rangle
$$


where $L=\mathbf{u} \cdot \nabla$ is an advection operator constructed with the smooth velocity field (note that $\mathbf{u}$ coincides with the field $\langle\mathbf{V}\rangle$ introduced previously). Subtracting Eq. (170) from Eq. (69) and neglecting non linear terms in the fluctuations, we obtain the following linearized equation for the evolution of the fluctuations 1

$$
\frac{\partial \delta \omega}{\partial t}+L \delta \omega=-\delta \mathbf{u} \nabla \omega
$$

Equations (70) and (71) form the basis of the quasilinear theory of the point vortex gas. These equations have been studied by Dubin \& O'Neil [20] in the case where the smooth vorticity profile is axisymmetric. In that case, Eq. (71) can be solved exactly with the aid of LaplaceFourier transforms and a kinetic equation which takes into account collective effects can be obtained. This is the counterpart of the quasilinear theory in plasma physics that is used to derive the Lenard-Balescu equation from the Klimontovich equation [44. In the present work, we shall proceed differently so as to treat the case of systems that are not necessarily axisymmetric and not necessarily Markovian. Our method avoids the use of Laplace-Fourier transforms and remains in physical space. This yields expressions with a clear interpretation which enlightens the basic physics. The drawback of our approach, however, is that it neglects collective effects. The formal solution of Eq. (71) is

$$
\delta \omega(t)=G(t, 0) \delta \omega(0)-\int_{0}^{t} d \tau G(t, t-\tau) \delta \mathbf{u}(t-\tau) \nabla \omega(t-\tau),
$$

where $G$ is the Green function associated with the advection operator $L$ corresponding to the smooth mean field and we have noted $\omega(t)=\omega(\mathbf{r}, t)$ and $\delta \mathbf{u}(t)=\delta \mathbf{u}(\mathbf{r}, t)$ for brevity. On the other hand, the perturbation of the velocity field is related to the perturbation of the vorticity through

$$
\delta \mathbf{u}(t)=\frac{1}{\gamma} \int \mathbf{V}(1 \rightarrow 0) \delta \omega_{1}(t) d \mathbf{r}_{1}
$$

where 0 refers to the position r. Therefore, considering Eqs. (172) and (173), we see that the velocity fluctuation $\delta \mathbf{u}(t)$ is given by an iterative process: $\delta \mathbf{u}(t)$ depends on $\delta \omega_{1}(t)$ which itself depends on $\delta \mathbf{u}_{1}(t-\tau)$ etc. We shall solve this problem perturbatively 2 in the thermodynamic

\footnotetext{
${ }^{1}$ As shown in Sec. 2.2, the proper thermodynamic limit corresponds to $N \rightarrow+\infty$ in such a way that the individual circulation $\gamma \sim 1 / N$ and the domain area $V \sim 1$. This implies that $|\mathbf{r}| \sim 1$. We also have $\omega \sim 1$ and $\delta \omega \sim 1 / \sqrt{N}$ so that $|\mathbf{u}| \sim 1$ and $\delta|\mathbf{u}| \sim 1 / \sqrt{N}$. With these scalings, we see that the terms that we have kept in Eq. (711) are of order $u \delta \omega \sim 1 / \sqrt{N}$ and $\omega \delta u \sim 1 / \sqrt{N}$ while the nonlinear terms that we have neglected are of order $\delta \omega \delta u \sim 1 / N \ll 1 / \sqrt{N}$. We also note that the l.h.s. of Eq. (70) is of order $\omega \sim 1$ while the r.h.s. of Eq. (70) is of order $\delta \omega \delta u \sim 1 / N$. Then Eq. (70) can be rewritten $\partial_{t} \omega+L \omega=(1 / N) C(\omega)$ where the advective term is of order $O(1)$ and the collision term is of order $1 / N$. Therefore, this equation describes the evolution of the system on a timescale $\sim N t_{D}$. For $N \rightarrow+\infty$, it reduces to the $2 \mathrm{D}$ Euler equation $\partial_{t} \omega+L \omega=0$. In conclusion, the quasilinear theory developed in this section is equivalent to the truncation of the BBGKY hierarchy at the order $1 / N$. It amounts to neglecting three-body and higher correlations.

${ }^{2}$ It is at that stage of the developement that we neglect some collective effects. In the approach of Dubin \& O'Neil [20] for axisymmetric flows, Eqs. (72) and (73) can be solved exactly.
} 
limit $N \rightarrow+\infty$ defined in Sec. 2.2. To order $1 / N$ we get

$$
\begin{array}{r}
\langle\delta \mathbf{u} \nabla \delta \omega\rangle=\frac{1}{\gamma} \frac{\partial}{\partial r^{\mu}} \int d \mathbf{r}_{1} V^{\mu}(1 \rightarrow 0) G_{1}(t, 0) G(t, 0)\left\langle\delta \omega_{1}(0) \delta \omega(0)\right\rangle \\
-\frac{1}{\gamma^{2}} \frac{\partial}{\partial r^{\mu}} \int_{0}^{t} d \tau \int d \mathbf{r}_{1} d \mathbf{r}_{2} V^{\mu}(1 \rightarrow 0) G_{1}(t, t-\tau) G(t, t-\tau) \\
\times\left\{V^{\nu}(2 \rightarrow 0)\left\langle\delta \omega_{1}(t-\tau) \delta \omega_{2}(t-\tau)\right\rangle \frac{\partial \omega}{\partial r^{\nu}}(t-\tau)\right. \\
\left.+V^{\nu}(2 \rightarrow 1)\left\langle\delta \omega(t-\tau) \delta \omega_{2}(t-\tau)\right\rangle \frac{\partial \omega_{1}}{\partial r_{1}^{\nu}}(t-\tau)\right\} .
\end{array}
$$

Now, the fluctuation is exactly defined by

$$
\delta \omega(\mathbf{r}, t)=\sum_{i} \gamma \delta\left(\mathbf{r}-\mathbf{r}_{i}(t)\right)-\omega(\mathbf{r}, t) .
$$

Therefore, we obtain

$$
\begin{array}{r}
\left\langle\delta \omega_{1} \delta \omega_{2}\right\rangle=\left\langle\sum_{i \neq j} \gamma^{2} \delta\left(\mathbf{r}_{1}-\mathbf{r}_{i}\right) \delta\left(\mathbf{r}_{2}-\mathbf{r}_{j}\right)\right\rangle+\left\langle\sum_{i} \gamma^{2} \delta\left(\mathbf{r}_{1}-\mathbf{r}_{i}\right) \delta\left(\mathbf{r}_{2}-\mathbf{r}_{i}\right)\right\rangle \\
-\left\langle\sum_{i} \gamma \delta\left(\mathbf{r}_{1}-\mathbf{r}_{i}\right) \omega_{2}\right\rangle-\left\langle\sum_{j} \gamma \delta\left(\mathbf{r}_{2}-\mathbf{r}_{j}\right) \omega_{1}\right\rangle+\omega_{1} \omega_{2} .
\end{array}
$$

To evaluate the correlation functions, we average with respect to the smooth distribution $\omega_{i} /(N \gamma)$ or $\omega_{i} \omega_{j} /(N \gamma)^{2}$. This operation leads to

$$
\left\langle\delta \omega_{1} \delta \omega_{2}\right\rangle=\frac{N-1}{N} \omega_{1} \omega_{2}+\gamma \omega_{1} \delta\left(\mathbf{r}_{1}-\mathbf{r}_{2}\right)-\omega_{1} \omega_{2}-\omega_{2} \omega_{1}+\omega_{1} \omega_{2},
$$

so that, finally,

$$
\left\langle\delta \omega_{1} \delta \omega_{2}\right\rangle=\gamma \omega_{1} \delta\left(\mathbf{r}_{1}-\mathbf{r}_{2}\right)-\frac{1}{N} \omega_{1} \omega_{2} .
$$

Substituting this result in Eq. (74), we obtain

$$
\begin{array}{r}
\langle\delta \mathbf{u} \nabla \delta \omega\rangle=\left\langle V^{\mu}(1 \rightarrow 0)\right\rangle \frac{\partial \omega}{\partial r^{\mu}}+\frac{1}{\gamma} \frac{\partial}{\partial r^{\mu}} \int_{0}^{t} d \tau \int d \mathbf{r}_{1} V^{\mu}(1 \rightarrow 0) G(t, t-\tau) \\
\left\{\mathcal{V}^{\nu}(1 \rightarrow 0) \omega_{1}(t-\tau) \frac{\partial \omega}{\partial r^{\nu}}(t-\tau)+\mathcal{V}^{\nu}(0 \rightarrow 1) \omega(t-\tau) \frac{\partial \omega_{1}}{\partial r_{1}^{\nu}}(t-\tau)\right\},
\end{array}
$$

where we have regrouped the two Greenians $G$ and $G_{1}$ in a single notation for brevity. Finally, replacing this expression in Eq. (70), we obtain the kinetic equation

$$
\begin{aligned}
& \frac{\partial \omega}{\partial t}+\frac{N-1}{N} \mathbf{u} \nabla \omega=\gamma \frac{\partial}{\partial r^{\mu}} \int_{0}^{t} d \tau \int d \mathbf{r}_{1} \frac{\mathcal{V}^{\mu}}{\gamma}(1 \rightarrow 0) G(t, t-\tau) \\
& \times\left\{\frac{\mathcal{V}^{\nu}}{\gamma}(1 \rightarrow 0) \omega_{1} \frac{\partial \omega}{\partial r^{\nu}}+\frac{\mathcal{V}^{\nu}}{\gamma}(0 \rightarrow 1) \omega \frac{\partial \omega_{1}}{\partial r_{1}^{\nu}}\right\}_{t-\tau} .
\end{aligned}
$$

This is identical to the general kinetic equation (52) obtained from the projection operator formalism or from the BBGKY-like hierarchy. We note that the term of order $1 / N$ in the l.h.s. comes from the first term in Eq. (72). It corresponds to the mere advection of the vorticity fluctuation by the smooth velocity field in Eq. (71), i.e. ignoring the coupling between the velocity fluctuations and the smooth vorticity (r.h.s. of Eq. (71)) which gives rise to the collision term. 


\subsection{The violent collisionless evolution of point vortices}

To leading order in $N \rightarrow+\infty$, the smooth vorticity profile of the point vortex gas is solution of the 2D Euler-Poisson system

$$
\frac{\partial \omega}{\partial t}+\mathbf{u} \nabla \omega=0, \quad \omega=-\Delta \psi
$$

This is the counterpart of the Vlasov-Poisson system in stellar dynamics and plasma physics. The 2D Euler equation describes the collisionless evolution of the point vortices due to mean field effects before collisions come into play on a timescale $N t_{D}$ or larger. Starting from an initial condition which is dynamically unstable, the 2D Euler-Poisson system develops an intricate filamentation at smaller and smaller scales. In this sense, the fine-grained vorticity $\omega(\mathbf{r}, t)$ never achieves equilibrium. However, if we locally average over the filaments, the resulting "coarse-grained" vorticity $\bar{\omega}(\mathbf{r}, t)$ will achieve a steady state on a timescale $\sim t_{D}[8$. Since the $2 \mathrm{D}$ Euler equation is only valid in the collisionless regime $t \ll t_{\text {coll }}$, this corresponds to a quasistationary state (QSS) that will slowly evolve due to the effect of collisions occuring on a longer timescale $\sim N t_{D}$ or larger. We can try to predict this QSS in terms of a statistical mechanics of the 2D Euler equation, using the approach of Miller-Robert-Sommeria [32, 33] 3. This is the 2D hydrodynamic version of the theory of violent relaxation proposed by Lynden-Bell [31] for collisionless stellar systems based on the Vlasov equation. In the case where the fine-grained vorticity $\omega(\mathbf{r}, t)$ takes only two values 0 and $\sigma_{0}$, the statistical equilibrium state maximizes the mixing entropy

$$
S_{M R S}=-\int\left\{\frac{\bar{\omega}}{\sigma_{0}} \ln \frac{\bar{\omega}}{\sigma_{0}}+\left(1-\frac{\bar{\omega}}{\sigma_{0}}\right) \ln \left(1-\frac{\bar{\omega}}{\sigma_{0}}\right)\right\} d \mathbf{r},
$$

at fixed circulation and energy. This leads to the coarse-grained vorticity

$$
\bar{\omega}=\frac{\sigma_{0}}{1+\lambda e^{\beta \sigma_{0} \psi}} .
$$

Note that the mixing entropy (82) is formally similar to the Fermi-Dirac entropy and the equilibrium distribution (83) is formally similar to the Fermi-Dirac distribution. An effective "exclusion principle", similar to the Pauli principle in quantum mechanics, arises in the theory of violent relaxation of continuous vorticity fields because the different levels of vorticity cannot overlap. We also note that the violent relaxation of point vortices does not lead to a segregation of the vortices according to their circulation (in the multi-species case) because the circulation of the individual vortices does not appear in the 2D Euler equation. Finally, we stress that the MRS theory is based on an assumption of ergodicity. Indeed, it implicitly assumes that the vorticity mixes well so that the QSS is the most mixed state compatible with the integral constraints of the 2D Euler equation. This may not always be the case as discussed in Sec. 4.3.

A kinetic theory of the process of violent relaxation has been developed in [30] with the aim to determine the dynamical equation satisfied by the coarse-grained vorticity field $\bar{\omega}(\mathbf{r}, t)$. This approach is based on a quasilinear approximation of the 2D Euler equation that is formally similar to that developed in the previous section (but with a completely different interpretation). In Sec. 4.1, the subdynamics was played by $\omega_{d}$ (a sum of $\delta$-functions) and the macrodynamics by $\omega$ (a smooth field). The smooth field averages over the positions of the $\delta$-functions (point vortices) that strongly fluctuate. In the phase of violent relaxation, the "smooth" field $\omega$

\footnotetext{
${ }^{3}$ In these works, the 2D Euler equation is justified as a limit of the Navier-Stokes equation for inviscid fluids $\nu \rightarrow 0$. Since the 2D Euler equation also describes the collisionless regime of the point vortex gas, their approach can be applied in that context.
} 
develops itself a finely striated structure and strongly fluctuates. Therefore, it is not smooth at a higher scale of resolution and a second smoothing procedure (coarse-graining) must be introduced. In that case, the subdynamics is played by $\omega$ and the macrodynamics by $\bar{\omega}$. The coarse-grained field averages over the positions of the filaments. The quasilinear theory leads to a kinetic equation for the coarse-grained vorticity of the form [30]:

$$
\begin{array}{r}
\frac{\partial \bar{\omega}}{\partial t}+\overline{\mathbf{u}} \nabla \bar{\omega}=\epsilon^{2} \frac{\partial}{\partial r^{\mu}} \int_{0}^{t} d \tau \int d \mathbf{r}_{1} \frac{V^{\mu}}{\gamma}(1 \rightarrow 0) G(t, t-\tau) \\
\times\left\{\frac{V^{\nu}}{\gamma}(1 \rightarrow 0) \bar{\omega}_{1}\left(\sigma_{0}-\bar{\omega}_{1}\right) \frac{\partial \bar{\omega}}{\partial r^{\nu}}+\frac{V^{\nu}}{\gamma}(0 \rightarrow 1) \bar{\omega}\left(\sigma_{0}-\bar{\omega}\right) \frac{\partial \bar{\omega}_{1}}{\partial r_{1}^{\nu}}\right\}_{t-\tau},
\end{array}
$$

where $\epsilon$ is the coarse-graining mesh size and $\overline{\mathbf{u}}$ the velocity field produced by the coarse-grained vorticity (recall also that the ratio $\mathbf{V}(1 \rightarrow 0) / \gamma$ only depends on $\mathbf{r}_{1}$ and $\mathbf{r}$ according to Eq. (5)). This equation is expected to describe the late quiescent stages of the relaxation process when the fluctuations have weaken so that the quasilinear approximation can be implemented. It does not describe the early, very chaotic, process of violent relaxation driven by the strong fluctuations of the stream function. The quasilinear theory of the 2D Euler equation is therefore a theory of "quiescent" collisionless relaxation. This is the counterpart of the quasilinear theory of the Vlasov-Poisson system developed for collisionless stellar systems [45, 46, 47].

Equation (84) is very similar, in structure, to Eq. (80) for the collisional evolution of point vortices, with nevertheless three important differences: (i) the fluctuating velocity $\mathcal{V}(1 \rightarrow 0)$ is replaced by the direct velocity $V(1 \rightarrow 0)$ because the fluctuations are taken into account differently. (ii) The vorticity $\omega$ in the collisional term of equation (80) is replaced by the product $\bar{\omega}\left(\sigma_{0}-\bar{\omega}\right)$ in equation (84). This nonlinear term arises from the effective "exclusion principle" accounting for the non-overlapping of vortex patches in the collisionless regime. This is consistent with the Fermi-Dirac-like entropy (82) and Fermi-Dirac-like distribution (83) at statistical equilibrium. (iii) Considering the dilute limit $\bar{\omega} \ll \sigma_{0}$ to fix the ideas, we see that the equations (84) and (80) have the same mathematical form differing only in the prefactors: the circulation $\gamma$ of a point vortex in Eq. (80) is replaced by the circulation $\sigma_{0} \epsilon^{2}$ of a completely filled macrocell in Eq. (84). This implies that the timescales of collisional and collisionless "relaxation" are in the ratio

$$
\frac{t_{\text {ncoll }}}{t_{\text {coll }}} \sim \frac{\gamma}{\sigma_{0} \epsilon^{2}}
$$

Since $\sigma_{0} \epsilon^{2} \gg \gamma$, this ratio is in general quite small implying that the collisionless relaxation is much more rapid than the collisional relaxation. Typically, $t_{n c o l l}$ is of the order of a few dynamical times $t_{D}$ (its precise value depends on the size of the mesh) while $t_{\text {coll }}$ is of order $N t_{D}$, or larger. The kinetic equation (84) conserves the circulation, the angular momentum and, presumably, the energy. By contrast, we cannot prove an $H$-theorem for the MRS entropy (82). Indeed, the time variation of the MRS entropy is of the form

$$
\begin{gathered}
\dot{S}_{M R S}=\frac{1}{2} \epsilon^{2} \int d \mathbf{r} d \mathbf{r}_{1} \frac{1}{\bar{\omega}\left(\sigma_{0}-\bar{\omega}\right) \bar{\omega}_{1}\left(\sigma_{0}-\bar{\omega}_{1}\right)} \int_{0}^{t} d \tau Q(t) G(t, t-\tau) Q(t-\tau), \\
Q(t)=\left[\frac{V^{\mu}}{\gamma}(1 \rightarrow 0) \bar{\omega}_{1}\left(\sigma_{0}-\bar{\omega}_{1}\right) \frac{\partial \bar{\omega}}{\partial r^{\mu}}+\frac{V^{\mu}}{\gamma}(0 \rightarrow 1) \bar{\omega}\left(\sigma_{0}-\bar{\omega}\right) \frac{\partial \bar{\omega}_{1}}{\partial r_{1}^{\mu}}\right],
\end{gathered}
$$

and its sign is not necessarily positive. This depends on the importance of memory effects. 


\subsection{Discussion: incomplete violent relaxation}

Even if Eq. (84) conserves the energy and the circulation and increases the MRS entropy (82) monotonically, this does not necessarily imply that the system will converge towards the MRS distribution (83). There are many cases where the MRS theory provides a good prediction of the QSS [48, 49]. However, it has also been observed in some experiments [50] and numerical simulations [51] that the QSS does not exactly coincide with the strict statistical equilibrium state predicted by the MRS theory because of the complicated problem of incomplete relaxation [52. This is usually explained by a lack of ergodicity or "incomplete mixing". Here, we try to be a little more precise by using the kinetic theory. There can be several reasons of incomplete relaxation:

(i) Absence of resonances: Very few is known concerning kinetic equations of the form of Eq. (84) and it is not clear whether the MRS distribution (83) is a stationary solution of that equation (and whether it is the only one). In order to simplify Eq. (84), we shall assume that the coarse-grained vorticity field is axisymmetric and that the correlations relax on a timescale which is much shorter than the typical time on which the coarse-grained vorticity field changes (Markovian approximation). Although this approximation was justified to describe the collisional relaxation of point vortices (because of the timescale separation between the dynamical time and the collision time), this approximation is not clear for the process of violent collisionless relaxation where memory terms can be important. However, with this approximation we can re-do the calculations of the previous sections and obtain a kinetic equation of the form

$\frac{\partial \bar{\omega}}{\partial t}=-\frac{\epsilon^{2}}{4 r} \frac{\partial}{\partial r} \int_{0}^{+\infty} r_{1} d r_{1} \ln \left[1-\left(\frac{r_{<}}{r_{>}}\right)^{2}\right] \delta\left(\Omega-\Omega_{1}\right)\left\{\frac{1}{r} \omega_{1}\left(\sigma_{0}-\omega_{1}\right) \frac{\partial \omega}{\partial r}-\frac{1}{r_{1}} \omega\left(\sigma_{0}-\omega\right) \frac{\partial \omega_{1}}{\partial r_{1}}\right\}$.

This equation conserves the circulation, the energy and the angular momentum and satisfies an H-theorem for the mixing entropy (82). However, as already discussed for the collisional evolution of the point vortices, it does not relax in general towards the statistical equilibrium state, here the MRS distribution (83) because of the absence of resonances. The same conclusion probably applies to the more general equation (84) and to the heuristic equation (148) of [23] which also conserve $E, \Gamma, L$ and increase the mixing entropy (82) but do not obligatory relax towards the MRS distribution (83). The system tries to approach the statistical equilibrium state (as indicated by the increase of the entropy) but may be trapped in a QSS that is different from the statistical prediction (83). This QSS is a steady solution of Eq. (84) which cancels individually the advective term (l.h.s.) and the effective collision term (r.h.s.). This determines a subclass of steady states of the 2D Euler equation (cancellation of the 1.h.s.) such that the complicated "turbulent" current $\mathbf{J}$ in the r.h.s. vanishes. This offers a large class of possible steady state solutions that can explain the deviation between the QSS and the MRS statistical equilibrium state (83) observed, in certain cases, in simulations and experiments of violent relaxation. One may argue that nonlinear terms are needed in the kinetic theory in order to obtain an equation that relaxes towards the statistical equilibrium distribution (83). In that case, we must develop a kinetic theory that goes beyond the quasilinear approximation.

(ii) Incomplete relaxation in space: The turbulent current $\mathbf{J}$ in Eq. (84) is driven by the fluctuations $\omega_{2} \equiv \overline{\tilde{\omega}^{2}}=\overline{\omega^{2}}-\bar{\omega}^{2}$ of the vorticity (generating the fluctuations $\delta \mathbf{u}$ of the velocity) [30]. In the "mixing region" where the fluctuations are strong, the vorticity tends to reach the MRS distribution (83). As we depart from the "mixing region", the fluctuations decay $\left(\omega_{2} \rightarrow 0\right)$ and the mixing is less and less efficient. In these regions, the system takes a long time to reach the MRS distribution (83) and, in practice, cannot attain it in the time available 
(see (iii)). In the two levels case, we have $\omega_{2}=\bar{\omega}\left(\sigma_{0}-\bar{\omega}\right)$. Therefore, the regions where $\bar{\omega} \rightarrow 0$ or $\bar{\omega} \rightarrow \sigma_{0}$ do not mix well (the diffusion current $\mathbf{J}$ is weak) and the observed vorticity can be sensibly different from the MRS distribution in these regions. This concerns essentially the core $\left(\bar{\omega} \rightarrow \sigma_{0}\right)$ and the tail $(\bar{\omega} \rightarrow 0)$ of the vorticity distribution. This result, derived from the kinetic theory, is consistent with what is observed in experiments [50, 51]: the core vorticity decreases less than predicted by the MRS statistical theory while the tail of the vorticity profile decreases more rapidly. For the same reason, it can also explain why the vorticity peak is remarkably well conserved during a merging process as observed in 2D decaying turbulence [53].

(iii) Incomplete relaxation in time: during violent relaxation, the system tends to approach the statistical equilibrium state (83). However, as it approaches equilibrium, the fluctuations of the velocity field, which are the engine of the evolution, become less and less effective to drive the relaxation. This is because the scale of the fluctuations becomes smaller and smaller as time goes on. This effect can be taken into account in the kinetic theory by considering that the correlation length $\epsilon(t)$ decreases with time so that, in the kinetic equation (84), the prefactor $\epsilon(t) \rightarrow 0$ rapidly. As a result, the "turbulent" current $\mathbf{J}$ in Eq. (84) can vanish before the system has reached the statistical equilibrium state (83). In that case, the system can be trapped in a QSS that is a steady solution of the 2D Euler equation different from the statistical prediction (83).

\section{Relaxation of a test vortex in a bath}

In this section, we study the relaxation of a test vortex in a bath of field vortices. Specifically, we consider a collection of $N$ point vortices at statistical equilibrium (thermal bath) and introduce a new vortex in the system. To leading order in $N \rightarrow+\infty$, the point vortex is advected by the mean flow. However, due to finite $N$ effects, the test vortex undergoes discrete interactions with the vortices of the bath and progressively acquires their distribution. We wish to study this stochastic process. The probability density $P(\mathbf{r}, t)$ of finding the test vortex in $\mathbf{r}$ at time $t$ is governed by a Fokker-Planck equation involving a term of diffusion and a term of drift. In our previous papers, we obtained these terms from a linear response theory [54] or from the projection operator formalism [23]. In the present work, we obtain these terms directly from the equations of motion and show how collective effects can be included in the theory in the case of axisymmetric flows. Our approach is also valid if the bath is made of a distribution of field vortices that evolves slowly, so that it can be assumed stationary on a timescale $(N / \ln N) t_{D}$ which is the typical relaxation time of the test vortex in the bath (see [29] for details).

\subsection{Diffusion coefficient}

The increment of the position of the test vortex between $t$ and $t-s$ due to the fluctuations of the velocity is

$$
\Delta r^{\mu}=\int_{t-s}^{t} \mathcal{V}^{\mu}\left(t^{\prime}\right) d t^{\prime}
$$

After standard calculations (see, e.g., Sec. 4.2 of [55]), the second moment of the increments of position can be rewritten in the form

$$
\left\langle\frac{\Delta r^{\mu} \Delta r^{\nu}}{2 s}\right\rangle=\frac{1}{s} \int_{0}^{s}(s+\tau)\left\langle\mathcal{V}^{\mu}(t) \mathcal{V}^{\nu}(t-\tau)\right\rangle d \tau
$$


We shall assume that the correlation function decreases more rapidly than $\tau^{-1}$. Then, taking the limit $s \rightarrow+\infty$, we find that the diffusion coefficient is given by the Kubo formula

$$
D^{\mu \nu}=\left\langle\frac{\Delta r^{\mu} \Delta r^{\nu}}{2 \Delta t}\right\rangle=\lim _{s \rightarrow+\infty}\left\langle\frac{\Delta r^{\mu} \Delta r^{\nu}}{2 s}\right\rangle=\int_{0}^{+\infty}\left\langle\mathcal{V}^{\mu}(t) \mathcal{V}^{\nu}(t-\tau)\right\rangle d \tau .
$$

On the other hand, after straightforward calculations (see, e.g., Sec. 4.1 of [55]), we obtain

$$
\begin{array}{r}
\left\langle\mathcal{V}^{\mu}(t) \mathcal{V}^{\nu}(t-\tau)\right\rangle=N\left\langle\mathcal{V}^{\mu}(1 \rightarrow 0, t) \mathcal{V}^{\nu}(1 \rightarrow 0, t-\tau)\right\rangle \\
=\int d \mathbf{r}_{1} \mathcal{V}^{\mu}(1 \rightarrow 0, t) \mathcal{V}^{\nu}(1 \rightarrow 0, t-\tau) \frac{\omega}{\gamma}\left(\mathbf{r}_{1}\right)
\end{array}
$$

Therefore, combining Eqs. (91) and (92), we get

$$
D^{\mu \nu}=\int_{0}^{+\infty} d \tau d \mathbf{r}_{1} \mathcal{V}^{\mu}(1 \rightarrow 0, t) \mathcal{V}^{\nu}(1 \rightarrow 0, t-\tau) \frac{\omega}{\gamma}\left(\mathbf{r}_{1}\right)
$$

For an axisymmetric system, the diffusion coefficient is given by

$$
D=\left\langle\frac{(\Delta r)^{2}}{2 \Delta t}\right\rangle=\lim _{s \rightarrow+\infty}\left\langle\frac{(\Delta r)^{2}}{2 s}\right\rangle=\int_{0}^{+\infty}\left\langle V_{r}(t) V_{r}(t-\tau)\right\rangle d \tau,
$$

leading to

$$
D=\int_{0}^{+\infty} d \tau \int_{0}^{2 \pi} d \theta_{1} \int_{0}^{+\infty} r_{1} d r_{1} V_{r}(1 \rightarrow 0, t) V_{r}(1 \rightarrow 0, t-\tau) \frac{\omega}{\gamma}\left(r_{1}\right) .
$$

If we neglect collective effects, the velocity created by a field vortex 1 on the test vortex 0 is given by (see Appendix $\mathrm{A}$ ):

$$
V_{r}(1 \rightarrow 0, t)=i \gamma \frac{1}{r} \sum_{m} m \hat{u}_{m} e^{i m\left(\theta-\theta_{1}\right)} .
$$

At time $t-\tau$, we have

$$
V_{r}(1 \rightarrow 0, t-\tau)=i \gamma \frac{1}{r} \sum_{m} m \hat{u}_{m} e^{i m\left(\theta(t-\tau)-\theta_{1}(t-\tau)\right)} .
$$

To leading order in $N \rightarrow+\infty$, the point vortices are advected by the mean field velocity so that $\theta_{i}(t-\tau)=\theta_{i}-\Omega\left(r_{i}\right) \tau$ and $r_{i}(t-\tau)=r_{i}$ where $r_{i}=r_{i}(t)$ and $\theta_{i}=\theta_{i}(t)$ denote their position at time $t$. Thus, we get

$$
V_{r}(1 \rightarrow 0, t-\tau)=i \gamma \frac{1}{r} \sum_{m} m \hat{u}_{m} e^{i m(\phi-\Delta \Omega \tau)} .
$$

Substituting this expression in Eq. (95) and carrying out the integrations on $\theta_{1}$ and $\tau$, we obtain after straightforward calculations

$$
D=\frac{2 \pi^{2} \gamma}{r^{2}} \int_{0}^{+\infty} r_{1} d r_{1} \chi\left(r, r_{1}\right) \delta(\Delta \Omega) \omega\left(r_{1}\right),
$$

where the function $\chi\left(r, r_{1}\right)$ is defined in Eq. (47). If the profile of angular velocity is monotonic, we can use $\delta(\Delta \Omega)=\delta\left(r-r_{1}\right) /\left|\Omega^{\prime}(r)\right|$ and we find that

$$
D(r)=2 \pi^{2} \gamma \frac{\chi(r, r)}{|\Sigma(r)|} \omega(r),
$$


where $\Sigma=r \Omega^{\prime}(r)$ is the local shear. For the potential (158), we have

$$
\chi(r, r)=\frac{1}{8 \pi^{2}} \sum_{m=1}^{+\infty} \frac{1}{m}=\frac{1}{8 \pi^{2}} \ln \Lambda,
$$

where $\ln \Lambda \equiv \sum_{m=1}^{+\infty} \frac{1}{m}$ is a logarithmically diverging Coulomb factor that has to be regularized. This leads to the following expression of the diffusion coefficient

$$
D(r)=\frac{\gamma}{4} \ln \Lambda \frac{1}{|\Sigma(r)|} \omega(r) .
$$

This expression, with the shear reduction, was derived in Chavanis [54, 23] and Dubin \& Jin [56] from the Kubo formula. Because of the divergence of the Coulomb factor, the value of the diffusion coefficient is dominated by the contribution of field vortices at radial distance $r_{1}=r$, justifying the local approximation made in [54]. Therefore, Eq. (102) gives the dominant term in the diffusion coefficient (99) of a test vortex even if the profile of angular velocity of the field vortices is non-monotonic. In practice, the Coulomb factor has to be regularized as discussed in detail in [56, 29]. It is then found that $\ln \Lambda$ scales with the number of particles like $\frac{1}{2} \ln N$ in the thermodynamic limit $N \rightarrow+\infty$, in agreement with the rough estimates in [54].

\subsection{Drift coefficient}

In addition to its diffusive motion, a test vortex immersed in a bath of field vortices with spatially inhomogeneous vorticity distribution undergoes a systematic drift. The drift corresponds to the response of the field vortices to the perturbation caused by the test vortex, as in a polarization process. The test vortex modifies the density distribution of the field vortices and the retroaction of this perturbation on the test vortex causes its drift. The expression of the drift can be derived from a linear response theory starting from the Liouville equation as done in [54]. In this section, we show that it can also be obtained from the Klimontovich equation. This will make a close connection with the quasilinear theory developed in Sec. 4 .

The introduction of a test vortex in a bath of field vortices modifies the vorticity profile $\omega(\mathbf{r}, t)$ of the bath. Since this perturbation is small, it can be described by the linearized Euler equation

$$
\frac{\partial \delta \omega}{\partial t}+L \delta \omega=-\delta \mathbf{u} \nabla \omega
$$

whose formal solution is

$$
\delta \omega(t)=-\int_{0}^{t} d \tau G(t, t-\tau) \delta \mathbf{u}(t-\tau) \nabla \omega(t-\tau)
$$

We have used the fact that, initially, $\delta \omega(0)=0$. On the other hand, the perturbation of the velocity field in $\mathbf{r}$ is given by

$$
\delta \mathbf{u}(\mathbf{r}, t)=\frac{1}{\gamma} \int \mathbf{V}(1 \rightarrow 0) \delta \omega_{1}(t) d \mathbf{r}_{1}+\int \mathcal{V}(1 \rightarrow 0) \delta\left(\mathbf{r}_{1}-\mathbf{r}_{P}(t)\right) d \mathbf{r}_{1} .
$$

The second term is the velocity created by the test vortex at position $\mathbf{r}_{P}(t)$ and the first term is the fluctuation of the velocity due to the perturbed density distribution of the field vortices. Substituting Eq. (104) in Eq. (105), we obtain

$$
\begin{array}{r}
\delta \mathbf{u}(\mathbf{r}, t)=-\frac{1}{\gamma} \int_{0}^{t} d \tau \int d \mathbf{r}_{1} \mathbf{V}(1 \rightarrow 0) \\
G_{1}(t, t-\tau) \delta u_{1}^{\nu}(t-\tau) \frac{\partial \omega_{1}}{\partial r_{1}^{\nu}}(t-\tau) \\
+\int \mathcal{V}(1 \rightarrow 0) \delta\left(\mathbf{r}_{1}-\mathbf{r}_{P}(t)\right) d \mathbf{r}_{1}
\end{array}
$$


This is an integral equation for $\delta \mathbf{u}(\mathbf{r}, t)$. For an axisymmetric flow, this equation can be solved exactly by using Laplace-Fourier transforms as done in Schecter \& Dubin [57]. In order to treat more general flows, we shall make an approximation which amounts to neglecting some collective effects. We solve Eq. (106) by an iterative process: we first neglect the first term in Eq. (106) keeping only the contribution of the test particle. Then, we substitute this value in the first term of the r.h.s of Eq. (106). This operation gives

$$
\begin{aligned}
& \delta \mathbf{u}(\mathbf{r}, t)=-\frac{1}{\gamma} \int_{0}^{t} d \tau \int d \mathbf{r}_{1} d \mathbf{r}_{2} \mathbf{V}(1 \rightarrow 0) G_{1}(t, t-\tau) \mathcal{V}^{\nu}(2 \rightarrow 1) \\
& \times \frac{\partial \omega_{1}}{\partial r_{1}^{\nu}}(t-\tau) \delta\left(\mathbf{r}_{2}-\mathbf{r}_{P}(t-\tau)\right)+\int \mathcal{V}(1 \rightarrow 0) \delta\left(\mathbf{r}_{1}-\mathbf{r}_{P}(t)\right) d \mathbf{r}_{1} .
\end{aligned}
$$

This quantity represents the fluctuation of the velocity field in $\mathbf{r}$ caused by the introduction of a test vortex in the system and taking into account the retroaction of the field vortices. If we evaluate this expression at the position $\mathbf{r}_{P}$ of the test vortex and subtract the second term (self-interaction), we obtain the drift experienced by the test vortex in response to the perturbation that it caused. Denoting finally by 0 the position of the test vortex, we find that its drift is given by

$$
V_{p o l}^{\mu}=-\frac{1}{\gamma} \int_{0}^{t} d \tau \int d \mathbf{r}_{1} V^{\mu}(1 \rightarrow 0, t) \mathcal{V}^{\nu}(0 \rightarrow 1, t-\tau) \frac{\partial \omega}{\partial r_{1}^{\nu}}\left(\mathbf{r}_{1}(t-\tau)\right) .
$$

For a thermal bath, where the distribution of the field vortices is given by $\omega\left(\mathbf{r}_{1}\right)=A e^{-\beta \gamma \psi\left(\mathbf{r}_{1}\right)}$, we obtain

$$
V_{p o l}^{\mu}=\beta \int_{0}^{t} d \tau \int d \mathbf{r}_{1} V^{\mu}(1 \rightarrow 0, t) \mathcal{V}(0 \rightarrow 1, t-\tau) \cdot \nabla \psi\left(\mathbf{r}_{1}(t-\tau)\right) \omega\left(\mathbf{r}_{1}\right),
$$

where we have used $\omega\left(\mathbf{r}_{1}(t-\tau)\right)=\omega\left(\mathbf{r}_{1}(t)\right)$ since $\omega$ is a stationary solution of the 2D Euler equation. This is equivalent to the result of the linear response theory based on the Liouville equation [54] but it is obtained here in a simpler manner. We can also obtain this result in a slightly different way. We approximate $\delta \mathbf{u}(\mathbf{r}, t)$ in Eq. (103) by the velocity $\mathcal{V}(P \rightarrow 0)$ created by the test vortex so that

$$
\frac{\partial \delta \omega}{\partial t}+L \delta \omega=-\mathcal{V}(P \rightarrow 0) \nabla \omega
$$

This equation can be solved with Green functions yielding

$$
\delta \omega(t)=-\int_{0}^{t} d \tau G(t, t-\tau) \mathcal{V}(P \rightarrow 0, t-\tau) \nabla \omega(t-\tau) .
$$

This represents the perturbation of the distribution of field vortices caused by the introduction of the test vortex in the system. This perturbation produces in turn a velocity which causes the drift of the test vortex (by retroaction). If we substitute Eq. (111) in the first part of Eq. (105) and evaluate this quantity at the position of the test vortex, we recover Eq. (108) for the drift (see also Appendix C).

If we now consider an axisymmetric distribution of field vortices, the expression of the drift becomes

$$
V_{r}^{p o l}=-\frac{1}{\gamma} \int_{0}^{t} d \tau \int_{0}^{2 \pi} d \theta_{1} \int_{0}^{+\infty} r_{1} d r_{1} V_{r}(1 \rightarrow 0, t) V_{r_{1}}(0 \rightarrow 1, t-\tau) \frac{d \omega}{d r}\left(r_{1}\right) .
$$


Using the identity (153) and taking the limit $t \rightarrow+\infty$, we get

$$
V_{r}^{p o l}=\frac{1}{\gamma} \int_{0}^{+\infty} d \tau \int_{0}^{2 \pi} d \theta_{1} \int_{0}^{+\infty} r_{1} d r_{1} \frac{r}{r_{1}} V_{r}(1 \rightarrow 0, t) V_{r}(1 \rightarrow 0, t-\tau) \frac{d \omega}{d r}\left(r_{1}\right) .
$$

This is a sort of generalized Kubo relation involving the gradient of the density profile instead of the density profile itself. The nice similarity in the expressions of the diffusion coefficient (95) and drift term (113) is worth mentioning. The integrals on $\theta_{1}$ and $\tau$ can be evaluated in the same manner as in Sec. 5.1 and we obtain

$$
V_{r}^{p o l}=\frac{2 \pi^{2} \gamma}{r} \int_{0}^{+\infty} d r_{1} \chi\left(r, r_{1}\right) \delta(\Delta \Omega) \frac{d \omega}{d r}\left(r_{1}\right) .
$$

Now, the drift of the test vortex is due not only to the polarization process but also to the variation of the diffusion coefficient with $r$. As a result, the complete expression of the drift is

$$
V_{r}^{\text {drift }} \equiv\left\langle\frac{\Delta r}{\Delta t}\right\rangle=\frac{\partial D}{\partial r}+V_{r}^{p o l}
$$

From Eqs. (99) and (114), we obtain

$$
V_{r}^{\text {drift }}=2 \pi^{2} \gamma \int_{0}^{+\infty} r r_{1} d r_{1} \omega\left(r_{1}\right)\left(\frac{1}{r} \frac{\partial}{\partial r}-\frac{1}{r_{1}} \frac{\partial}{\partial r_{1}}\right) \chi\left(r, r_{1}\right) \delta(\Delta \Omega) \frac{1}{r^{2}},
$$

where we have used an integration by parts in Eq. (114). Expressions (99) and (116) for the diffusion coefficient and the drift term can also be obtained directly from the Hamiltonian equations, by making a systematic expansion of the trajectory of the point vortices in powers of $1 / N$ in the limit $N \rightarrow+\infty$ as shown in Appendix $\mathrm{C}$ of [29].

For a thermal bath, corresponding to the case where the field vortices are at statistical equilibrium, the vorticity profile is given by the Boltzmann distribution

$$
\omega\left(r_{1}\right)=A e^{-\beta \gamma \psi^{\prime}\left(r_{1}\right)},
$$

where $\psi^{\prime}=\psi+(1 / 2) \Omega_{L} r^{2}$ is the relative stream function. Then, we have

$$
\frac{d \omega_{1}}{d r_{1}}=-\beta \gamma \omega_{1} \frac{d \psi_{1}^{\prime}}{d r_{1}}=\beta \gamma \omega_{1}\left(\Omega\left(r_{1}\right)-\Omega_{L}\right) r_{1} .
$$

Substituting this relation in Eq. (114), using the $\delta$-function to replace $\Omega\left(r_{1}\right)$ by $\Omega(r)$, using $d \psi^{\prime} / d r=\left(-\Omega(r)+\Omega_{L}\right) r$ and comparing the resulting expression with Eq. (99), we finally find that

$$
V_{r}^{p o l}=-\beta \gamma D \frac{d \psi^{\prime}}{d r}
$$

The drift is perpendicular to the relative mean field velocity and the drift coefficient (mobility) is given by a sort of Einstein relation $\xi=D \beta \gamma$. We note that the drift coefficient and the diffusion coefficient depend on the position and we recall that the temperature is negative in cases of physical interest [16]. We also emphasize that the Einstein relation is valid for the drift $V_{r}^{\text {pol }}$ due to the polarization only, not for the total drift (116). We do not have this subtlety for the usual Brownian motion where the diffusion coefficient is constant.

If we now consider a bath with a monotonic profile of angular velocity, using the same arguments as in Sec. 5.1, we find that Eq. (114) reduces to

$$
V_{r}^{p o l}=2 \pi^{2} \gamma \frac{\chi(r, r)}{|\Sigma(r)|} \frac{d \omega}{d r}(r) .
$$


For the potential (158), using Eq. (101), we find that

$$
V_{r}^{p o l}=\frac{\gamma}{4} \ln \Lambda \frac{1}{|\Sigma(r)|} \frac{d \omega}{d r}(r)
$$

Due to the diverging factor $\ln \Lambda \sim \frac{1}{2} \ln N$, this expression also gives the dominant term of the drift in the case where the vorticity profile is non monotonic. Comparing with Eq. (102), we find that the drift velocity is related to the diffusion coefficient by the relation

$$
V_{r}^{p o l}=D \frac{d \ln \omega}{d r}
$$

This expression generalizes Eq. (119) for a bath that is out-of-equilibrium.

\subsection{Collective effects}

As explained previously, one specificity of our approach is to develop a formalism that allows to describe flows that are not necessarily axisymmetric. However, its main drawback is to ignore collective effects. In the case of axisymmetric flows, these collective effects can be taken into account as in the study of Schecter \& Dubin [57]. In this section, we briefly indicate how the preceding results can be generalized to account for these collective effects.

For axisymmetric flows, Eqs. (103) and (105) can be written (we consider here the usual situation where the potential of interaction between vortices is solution of the Poisson equation):

$$
\begin{gathered}
\frac{\partial \delta \omega}{\partial t}+\Omega(r, t) \frac{\partial \delta \omega}{\partial \theta}+\frac{1}{r} \frac{\partial \delta \psi}{\partial \theta} \frac{\partial \omega}{\partial r}=0 \\
{\left[\frac{\partial^{2}}{\partial r^{2}}+\frac{1}{r} \frac{\partial}{\partial r}+\frac{1}{r^{2}} \frac{\partial}{\partial \theta^{2}}\right] \delta \psi=-\delta \omega-\gamma \frac{1}{r} \delta\left(r-r_{P}(t)\right) \delta\left(\theta-\theta_{P}(t)\right)}
\end{gathered}
$$

where $\left(r_{P}, \theta_{P}\right)$ are the coordinates of the test vortex. These equations can be solved by taking the Laplace-Fourier transform of $\delta \omega$ and $\delta \psi$. Returning to physical space, the perturbed stream function can finally be written [57]:

$$
\delta \psi(r, \theta, t)=\gamma \sum_{m} e^{i m\left(\theta-\theta_{P}\right)} \hat{U}_{m}\left(r, r_{P}\right)
$$

where

$$
\hat{U}_{m}\left(r, r_{P}\right)=-\frac{1}{4 \pi^{2} i} \int_{\alpha-i \infty}^{\alpha+i \infty} \frac{G\left(r, r_{P}, m, s\right)}{s} e^{s t} d s
$$

where $G$ is the Green function solution of

$$
\left[\frac{\partial^{2}}{\partial r^{2}}+\frac{1}{r} \frac{\partial}{\partial r}-\frac{m^{2}}{r^{2}}-\frac{i m}{s+i m \Omega(r)} \frac{1}{r} \frac{\partial \omega}{\partial r}\right] G\left(r, r_{P}, m, s\right)=\frac{\delta\left(r-r_{P}\right)}{r} .
$$

Therefore, when we take into account collective effects, the radial velocity created by point vortex 1 on point vortex 0 (say) is given by

$$
V_{r}(1 \rightarrow 0)=\frac{\gamma}{r} \sum_{m} i m e^{i m\left(\theta-\theta_{1}\right)} \hat{U}_{m}\left(r, r_{1}\right) .
$$


Neglecting collective effects amounts to neglecting the last term in brackets in Eq. (127). It then reduces to the usual Poisson equation where the vorticity field is due to a single point vortex. Then, $G$ is equal to $G_{b a r e}=-2 \pi \hat{u}_{m}\left(r, r_{P}\right)$ so that $\hat{U}_{m}\left(r, r_{1}\right)$ is replaced by $\hat{u}_{m}\left(r, r_{1}\right)$ in Eq. (128). This returns the bare velocity (96) created by point vortex 1 on point vortex 0 .

In the computation of the diffusion coefficient, we can take into account collective effects by replacing $\hat{u}_{m}$ by $\hat{U}_{m}$ in Eq. (98). This yields Eqs. (99) and (100) where $\chi\left(r, r_{1}\right)$ is replaced by

$$
\chi\left(r, r_{1}\right)=\sum_{m}|m|\left|\hat{U}_{m}\left(r, r_{1}\right)\right|^{2} .
$$

In fact, for $r=r_{1}$, the series diverges for large $m$ indicating that the main contribution to the diffusion coefficient is due to close interactions, justifying a local approximation. We thus qualitativelty understand that collective effects will be negligible. For large $m$, we can replace $\left|\hat{U}_{m}(r, r)\right|^{2}$ by $\left|\hat{u}_{m}(r, r)\right|^{2}$ returning the result (102).

In the computation of the drift, we can take into account collective effects as follows. The velocity created in 0 by the introduction of the test vortex is

$$
V_{r}(P \rightarrow 0)=\frac{\gamma}{r} \sum_{m} i m \hat{U}_{m}\left(r, r_{P}\right) e^{i m\left(\theta-\theta_{P}\right)}
$$

The bare velocity due to the test vortex is

$$
V_{r}(P \rightarrow 0)=\frac{\gamma}{r} \sum_{m} i m \hat{u}_{m}\left(r, r_{P}\right) e^{i m\left(\theta-\theta_{P}\right)} .
$$

If we subtract Eq. (131) from Eq. (130), we get the velocity created in 0 by the perturbation of the distribution of the field vortices caused by the introduction of the test vortex. Evaluating this velocity at the position of the test vortex, we obtain the drift experienced by the test vortex due to the polarization process

$$
V_{r}^{p o l}=\frac{\gamma}{r} \sum_{m} i m\left(\hat{U}_{m}(r, r)-\hat{u}_{m}(r, r)\right) .
$$

This can also be written

$$
V_{r}^{p o l}=-\frac{\gamma}{r} \sum_{m} m \operatorname{Im}\left[\hat{U}_{m}(r, r)\right]
$$

The series diverges for $m \rightarrow+\infty$. If we replace $\operatorname{Im}\left[\hat{U}_{m}(r, r)\right]$ by its asymptotic behaviour for large $m$ [57], this returns Eq. (120).

Finally, we conclude that, concerning the evaluation of the diffusion coefficient and drift term, collective effects play a negligible role since these quantities are dominated by close interactions. This gives further justification to the approaches developed in Secs. 5.1 and 5.2 .

\subsection{The Fokker-Planck equation}

Assuming that the evolution is axisymmetric, the probability density $P(\mathbf{r}, t)=P(r, t)$ of finding the test vortex in $\mathbf{r}$ at time $t$ is governed by a Fokker-Planck equation of the form

$$
\frac{\partial P}{\partial t}=\frac{1}{2 r} \frac{\partial}{\partial r}\left[r \frac{\partial}{\partial r}\left(\frac{\left\langle(\Delta r)^{2}\right\rangle}{\Delta t} P\right)\right]-\frac{1}{r} \frac{\partial}{\partial r}\left(r P \frac{\langle\Delta r\rangle}{\Delta t}\right) .
$$

This Fokker-Planck approach assumes that the stochastic process is markovian which is a good approximation in our case, as we have already indicated. It also assumes that the higher order 
moments of the increment of radial position $\Delta r$ play a negligible role. This is indeed the case in the $N \rightarrow+\infty$ limit that we consider since they are of order $O\left(N^{-2}\right)$ or smaller. At order $O\left(N^{-1}\right)$, we have found that the second (diffusion) and first (drift) moments of the radial increments of position of the test vortex are given by

$$
\frac{\left\langle(\Delta r)^{2}\right\rangle}{2 \Delta t}=D, \quad \frac{\langle\Delta r\rangle}{\Delta t}=\frac{\partial D}{\partial r}+\eta
$$

with

$$
\begin{gathered}
D=\frac{2 \pi^{2} \gamma}{r^{2}} \int_{0}^{+\infty} r_{1} d r_{1} \chi\left(r, r_{1}\right) \delta(\Delta \Omega) \omega\left(r_{1}\right), \\
\eta \equiv V_{r}^{p o l}=\frac{2 \pi^{2} \gamma}{r} \int_{0}^{+\infty} d r_{1} \chi\left(r, r_{1}\right) \delta(\Delta \Omega) \frac{d \omega}{d r}\left(r_{1}\right) .
\end{gathered}
$$

The Fokker-Planck equation (134) can be written in the alternative form

$$
\frac{\partial P}{\partial t}=\frac{1}{r} \frac{\partial}{\partial r}\left[r\left(D \frac{\partial P}{\partial r}-P \eta\right)\right] .
$$

The two expressions (134) and (138) have their own interest. The expression (134) where the diffusion coefficient is placed after the second derivative $\partial^{2}(D P)$ involves the total drift $V_{r}^{\text {drift }}=\langle\Delta r\rangle / \Delta t$ and the expression (138) where the diffusion coefficient is placed between the derivatives $\partial D \partial P$ isolates the part of the drift $\eta=V_{r}^{\text {polar }}$ due to the polarization. This alternative form (138) has therefore a clear physical interpretation. Inserting the expressions (136) and (137) of the diffusion coefficient and drift term in Eq. (138), we obtain

$$
\frac{\partial P}{\partial t}=2 \pi^{2} \gamma \frac{1}{r} \frac{\partial}{\partial r} \int_{0}^{+\infty} r_{1} d r_{1} \chi\left(r, r_{1}\right) \delta\left(\Omega-\Omega_{1}\right)\left(\frac{1}{r} \frac{\partial}{\partial r}-\frac{1}{r_{1}} \frac{\partial}{\partial r_{1}}\right) P(r, t) \omega\left(r_{1}\right) .
$$

For a thermal bath, using Eq. (119), the Fokker-Planck equation (138) can be written

$$
\frac{\partial P}{\partial t}=\frac{1}{r} \frac{\partial}{\partial r}\left[r D(r)\left(\frac{\partial P}{\partial r}+\beta \gamma P \frac{d \psi^{\prime}}{d r}\right)\right]
$$

where $D(r)$ is given by Eq. (136). For a steady bath with a monotonic vorticity profile, using Eq. (122), the Fokker-Planck equation (138) can be written

$$
\frac{\partial P}{\partial t}=\frac{1}{r} \frac{\partial}{\partial r}\left[r D(r)\left(\frac{\partial P}{\partial r}-P \frac{d \ln \omega}{d r}\right)\right]
$$

where $D(r)$ is given by Eq. (102). These Fokker-Planck equations have been studied in detail by Chavanis \& Lemou [29] for different types of bath distribution. The distribution of the test vortex relaxes to the distribution of the bath on a typical timescale $(N / \ln N) t_{D}$ but the relaxation process is very peculiar and differs from the usual exponential relaxation. In particular, the evolution of the front profile in the tail of the distribution is very slow (logarithmic) and the temporal correlation function $\langle r(0) r(t)\rangle$ decreases like $\ln t / t$ (for a thermal bath). This is due to the rapid decay of the diffusion coefficient $D(r)$, like in the HMF model [41].

In our previous papers, we have obtained Eq. (139) directly from the projection operator formalism (see Sec. 4.1 of [29]). This amounts, in the kinetic equation (46), to replacing $\omega(r, t)$ by the distribution $P(r, t)$ of the test vortex and $\omega\left(r_{1}, t\right)$ by the static distribution $\omega\left(r_{1}\right)$ of the 
field vortices. This procedure transforms an integrodifferential equation (46) into a differential equation (139). Then, the expressions (135)-(137) of the diffusion and drift terms were obtained by identifying Eq. (139) with the Fokker-Planck equation (134). In the present paper, we have proceeded the other way round by first determining the moments (135)-(137) in Secs. 5.1 and 5.2, then inserting them in the Fokker-Planck equation (134). Although this procedure may appear more logical in some sense, the other approach based on the projection operator formalism is more powerful because it allows one to obtain more general equations that are non markovian and that relax the hypothesis of axisymmetry as discussed in the next section.

\subsection{More general kinetic equations}

It is instructive to compare the Fokker-Planck equation (139) with the more general equation obtained from the projection operator formalism

$$
\begin{aligned}
& \frac{\partial P}{\partial t}+\langle\mathbf{V}\rangle \nabla P=\frac{\partial}{\partial r^{\mu}} \int_{0}^{t} d \tau \int d \mathbf{r}_{1} V^{\mu}(1 \rightarrow 0) G(t, t-\tau) \\
& \times\left\{\mathcal{V}^{\nu}(1 \rightarrow 0) \frac{\partial}{\partial r^{\nu}}+\mathcal{V}^{\nu}(0 \rightarrow 1) \frac{\partial}{\partial r_{1}^{\nu}}\right\} P(\mathbf{r}, t-\tau) \frac{\omega}{\gamma}\left(\mathbf{r}_{1}\right)
\end{aligned}
$$

This equation can be obtained from Eq. (53), by replacing $\omega(r, t)$ by $P(r, t)$ and $\omega\left(r_{1}, t\right)$ by $\omega\left(r_{1}\right)$. This is a sort of generalized "Fokker-Planck" equation involving a term of "diffusion" and a term of "friction". However, strictly speaking, Eq. (142) is not a Fokker-Planck equation because it is non-Markovian. We also note that the "diffusion" term appears as a complicated time integral of the velocity correlation function involving $P(\mathbf{r}, t-\tau)$. This can be seen as a generalization of the Kubo formula (93). Similarly the "drift" is a generalization of the expression obtained in (108) with a more complicated time integral. If we consider a thermal bath where the distribution of the field vortices is the Boltzmann distribution, we get

$$
\begin{array}{r}
\frac{\partial P}{\partial t}+\langle\mathbf{V}\rangle \nabla P=\frac{\partial}{\partial r^{\mu}} \int_{0}^{t} d \tau \int d \mathbf{r}_{1} V^{\mu}(1 \rightarrow 0) G(t, t-\tau) \\
\times\left\{\mathcal{V}(1 \rightarrow 0) \cdot \nabla-\beta \gamma \mathcal{V}(0 \rightarrow 1) \cdot \nabla \psi\left(\mathbf{r}_{1}\right)\right\} P(\mathbf{r}, t-\tau) \frac{\omega}{\gamma}\left(\mathbf{r}_{1}\right) .
\end{array}
$$

If we come back to Eq. (142), make a Markovian approximation and extend the time integral to infinity, we obtain

$$
\begin{aligned}
\frac{\partial P}{\partial t}+\langle\mathbf{V}\rangle \nabla P=\frac{\partial}{\partial r^{\mu}} \int_{0}^{+\infty} d \tau \int d \mathbf{r}_{1} V^{\mu}(1 \rightarrow 0) G(t, t-\tau) \\
\times\left\{\mathcal{V}^{\nu}(1 \rightarrow 0) \frac{\partial}{\partial r^{\nu}}+\mathcal{V}^{\nu}(0 \rightarrow 1) \frac{\partial}{\partial r_{1}^{\nu}}\right\} P(\mathbf{r}, t) \frac{\omega}{\gamma}\left(\mathbf{r}_{1}\right),
\end{aligned}
$$

where we recall that the coordinates appearing after the Greenian must be viewed as explicit functions of time, i.e. $\mathbf{r}_{i}=\mathbf{r}_{i}(t-\tau)$. On the other hand, for an axisymmetric system, using the relation (153) and $r_{i}(t-\tau)=r_{i}(t)$ and $\theta_{i}(t-\tau)=\theta_{i}(t)-\Omega\left(r_{i}(t)\right) \tau$, Eq. (142) takes the simplest form

$$
\begin{aligned}
& \frac{\partial P}{\partial t}=\frac{1}{r} \frac{\partial}{\partial r} r \int_{0}^{t} d \tau \int_{0}^{2 \pi} d \theta_{1} \int_{0}^{+\infty} r r_{1} d r_{1} V_{r}(1 \rightarrow 0, t) \\
& \times V_{r}(1 \rightarrow 0, t-\tau)\left(\frac{1}{r} \frac{\partial}{\partial r}-\frac{1}{r_{1}} \frac{\partial}{\partial r_{1}}\right) P(r, t-\tau) \frac{\omega}{\gamma}\left(r_{1}\right) .
\end{aligned}
$$


If we make a Markovian approximation $P(r, t-\tau) \simeq P(r, t)$ and extend the time integral to infinity, we get

$$
\begin{aligned}
\frac{\partial P}{\partial t}= & \frac{1}{r} \frac{\partial}{\partial r} r \int_{0}^{+\infty} d \tau \int_{0}^{2 \pi} d \theta_{1} \int_{0}^{+\infty} r r_{1} d r_{1} V_{r}(1 \rightarrow 0, t) \\
& \times V_{r}(1 \rightarrow 0, t-\tau)\left(\frac{1}{r} \frac{\partial}{\partial r}-\frac{1}{r_{1}} \frac{\partial}{\partial r_{1}}\right) P(r, t) \frac{\omega}{\gamma}\left(r_{1}\right) .
\end{aligned}
$$

This is a Fokker-Planck equation which can be put in the form (138) with a diffusion coefficient

$$
D=\int_{0}^{+\infty} d \tau \int_{0}^{2 \pi} d \theta_{1} \int_{0}^{+\infty} r_{1} d r_{1} V_{r}(1 \rightarrow 0, t) V_{r}(1 \rightarrow 0, t-\tau) \frac{\omega}{\gamma}\left(r_{1}\right)
$$

and a drift term due to the polarization

$$
\eta=\frac{1}{\gamma} \int_{0}^{+\infty} d \tau \int_{0}^{2 \pi} d \theta_{1} \int_{0}^{+\infty} r d r_{1} V_{r}(1 \rightarrow 0) V_{r}(1 \rightarrow 0, t-\tau) \frac{d \omega}{d r}\left(r_{1}\right)
$$

These expressions agree with Eqs. (95) and (113) obtained directly from the equations of motion. After integration on $\tau$ and $\theta_{1}$, we recover the Fokker-Planck equation (139) with the expressions (136) and (137) of the diffusion coefficient and drift term.

\section{Conclusion}

In this paper, we have developed the kinetic theory of point vortices in two-dimensional hydrodynamics initiated in [23]. Point vortices provide a fundamental example of systems with long-range interactions [8] which deserves a particular attention. We have shown that the main features of the kinetic theory: kinetic equation describing the evolution of the system as a whole, diffusion coefficient, drift term, Fokker-Planck equation describing the evolution of a test particle in a bath... could be obtained from a simpler formalism than the one developed in our previous papers [54, 23]. This clarifies the argumentation and delineates the domain of validity of the theory. We have given general equations that are valid for flows that are not necessarily axisymmetric nor markovian. A limitation of our approach is to neglect collective effects. These effects have been taken into account in [20, 57] for axisymmetric flows. In plasma physics, collective effects are important because they lead to Debye shielding and regularize the logarithmic divergence at large scales that appears in the Landau equation (as shown by Lenard [21] and Balescu [22]). For point vortices, their influence seems less crucial since the kinetic equation (48) derived by neglecting collective terms does not present any divergence. In addition, concerning the expressions of the diffusion coefficient and drift term, we have indicated that collective effects have a negligible contribution because the diffusion coefficient and the drift velocity are dominated by local interactions. In future works, we plan to study in more detail the kinetic equations given in this paper. This project has been initiated in [29]. The kinetic theory could be used to interprete the numerical simulations of point vortices in $2 \mathrm{D}$ hydrodynamics [58, 59] or the experiments of non-neutral plasmas under a strong magnetic field (leading to quasi stationary states, vortex crystals,...) [60]. In agreement with the kinetic theory, these systems exhibit a violent collisionless relaxation and a slow collisional evolution. The collisionless relaxation is based on the 2D Euler equation and the evolution of the coarsegrained vorticity is described by Eq. (84). The collisional evolution was the main object of interest of the present paper. It is described by a general kinetic equation of the form (52) that simplifies in Eq. (48) for axisymmetric flows. 


\section{A The potential of interaction}

The velocity of the $i$-th vortex is produced by the other vortices according to the relation

$$
\mathbf{V}_{i}=-\frac{1}{\gamma} \mathbf{z} \times \nabla_{i} H=\sum_{j \neq i} \mathbf{V}(j \rightarrow i),
$$

where $H$ is the Hamiltonian (2). The velocity created by point vortex $j$ on point vortex $i$ is

$$
\mathbf{V}(j \rightarrow i)=-\gamma \mathbf{z} \times \frac{\partial u_{i j}}{\partial \mathbf{r}_{i}}
$$

Introducing a system of polar coordinates to localize the point vortices, the radial component in the direction of $\mathbf{r}_{1}$ of the velocity created by point vortex 2 on point vortex 1 is

$$
V_{r_{1}}(2 \rightarrow 1)=\frac{\gamma}{r_{1}} \frac{\partial u_{12}}{\partial \theta_{1}}
$$

In an infinite domain, the potential of interaction can be written

$$
u_{12}=u\left(\sqrt{r_{1}^{2}+r_{2}^{2}-2 r_{1} r_{2} \cos \phi}\right) \equiv u\left(r_{1}, r_{2}, \phi\right),
$$

where $\phi=\theta_{1}-\theta_{2}$. This implies that

$$
V_{r_{2}}(1 \rightarrow 2)=-\frac{r_{1}}{r_{2}} V_{r_{1}}(2 \rightarrow 1) .
$$

This relation results from the conservation of the angular momentum (see Appendix D of [23])

and remains valid in a bounded circular domain. Since the function $u\left(r_{1}, r_{2}, \phi\right)$ is periodic with period $2 \pi$, it can be decomposed in Fourier series. Thus,

$$
u\left(r_{1}, r_{2}, \phi\right)=\sum_{m} e^{i m \phi} \hat{u}_{m}\left(r_{1}, r_{2}\right),
$$

with

$$
\hat{u}_{m}\left(r_{1}, r_{2}\right)=\frac{1}{2 \pi} \int_{0}^{2 \pi} \cos (n \phi) u\left(r_{1}, r_{2}, \phi\right) d \phi .
$$

Using the decomposition (154), we find that Eq. (151) can be rewritten in the form

$$
V_{r_{1}}(2 \rightarrow 1)=i \gamma \frac{1}{r_{1}} \sum_{m} m \hat{u}_{m}\left(r_{1}, r_{2}\right) e^{i m\left(\theta_{1}-\theta_{2}\right)}
$$

The usual potential of interaction between point vortices is solution of the Poisson equation

$$
\Delta u=-\delta(\mathbf{r})
$$

In an infinite domain, we have

$$
u_{12}=-\frac{1}{2 \pi} \ln \left|\mathbf{r}_{1}-\mathbf{r}_{2}\right|
$$

The Fourier transform of $u(\phi)$ can be easily obtained by using, e.g., the identities given in Appendix E1 of [23]. We find

$$
\hat{u}_{m}\left(r_{1}, r_{2}\right)=\frac{1}{4 \pi|m|}\left(\frac{r_{<}}{r_{>}}\right)^{|m|}, \quad(m \neq 0)
$$




$$
\hat{u}_{0}\left(r_{1}, r_{2}\right)=-\frac{1}{2 \pi} \ln r_{>}
$$

where $r_{>}$(resp. $r_{<}$) is the largest (resp. smallest) of $r_{1}$ and $r_{2}$. In that case, the function defined in Eq. (47) takes the explicit form

$$
\chi\left(r_{1}, r_{2}\right)=\frac{1}{8 \pi^{2}} \sum_{m=1}^{+\infty} \frac{1}{m}\left(\frac{r_{<}}{r_{>}}\right)^{2 m}=-\frac{1}{8 \pi^{2}} \ln \left[1-\left(\frac{r_{<}}{r_{>}}\right)^{2}\right] .
$$

When the point vortices are confined within a circular box of radius $R$, the potential of interaction is

$$
u_{12}=-\frac{1}{2 \pi} \ln \left|\mathbf{r}_{1}-\mathbf{r}_{2}\right|+\frac{1}{2 \pi} \ln \left|\mathbf{r}_{1}-\frac{R^{2}}{r_{2}^{2}} \mathbf{r}_{2}\right|
$$

and its Fourier transform is given by

$$
\begin{gathered}
\hat{u}_{m}=\frac{1}{4 \pi|m|}\left(\frac{r_{<}}{r_{>}}\right)^{|m|}\left[1-\left(\frac{r_{>}}{R}\right)^{2|m|}\right], \quad(m \neq 0) \\
\hat{u}_{0}\left(r_{1}, r_{2}\right)=\frac{1}{2 \pi} \ln \left(\frac{R^{2}}{r_{2} r_{>}}\right) .
\end{gathered}
$$

In that case, the function defined in Eq. (477) takes the explicit form

$$
\chi\left(r_{1}, r_{2}\right)=-\frac{1}{8 \pi^{2}} \ln \left[1-\left(\frac{r_{<}}{r_{>}}\right)^{2}\right]+\frac{1}{4 \pi^{2}} \ln \left[1-\left(\frac{r_{<}}{R}\right)^{2}\right]-\frac{1}{8 \pi^{2}} \ln \left[1-\left(\frac{r_{<} r_{>}}{R^{2}}\right)^{2}\right] .
$$

Finally, in the QG model, the potential of interaction is solution of an equation of the form

$$
\Delta u-\frac{1}{L^{2}} u=-\delta(\mathbf{r})
$$

where $L$ is the Rossby radius. In an infinite domain, we obtain

$$
u_{12}=\frac{1}{2 \pi} K_{0}\left(\frac{\left|\mathbf{r}_{1}-\mathbf{r}_{2}\right|}{L}\right)
$$

\section{B A heuristic kinetic equation}

In [23] and in Sec. 3.3, we have derived a general kinetic equation (52) or (54) that is valid at order $O(1 / N)$. This equation can be simplified for axisymmetric flows (leading to Eq. (48)) and uni-directional flows (leading to Eq. (135) of [23]). In [23], we have heuristically proposed a generalized kinetic equation (137) that encompasses both the axisymmetric and unidirectional forms. This equation is not exact, so it cannot be obtained rigorously from Eq. (52). Yet, it possesses interesting properties (conservation of $E, \Gamma, L, P$ and $H$-theorem $\dot{S} \geq 0$ ) so it can be useful. For axisymmetric and unidirectional flows, it does not exactly reduce to Eq. (48) and Eq. (135) of [23], but it has a similar structure so that the disagreement is not too severe. In this Appendix, we try to justify this equation but we stress that, since this equation is not exact, some approximations are necessarily un-controlled. 
First, assuming that the decorrelation time is extremely short (which does not need to be the case) and that $\mathcal{V} \simeq \mathbf{V}$, we replace Eq. (54) by

$$
\begin{aligned}
\frac{\partial \omega_{1}}{\partial t}+\frac{N-1}{N}\langle\mathbf{V}\rangle_{1} \frac{\partial \omega}{\partial \mathbf{r}_{1}}=\frac{\partial}{\partial r_{1}^{\mu}} \int_{0}^{+\infty} d \tau \int & d \mathbf{r}_{2} V^{\mu}(2 \rightarrow 1, t) V^{\nu}(2 \rightarrow 1, t-\tau) \\
& \times\left(\frac{\partial}{\partial r_{1}^{\nu}}-\frac{\partial}{\partial r_{2}^{\nu}}\right) \omega\left(\mathbf{r}_{1}, t\right) \frac{\omega}{\gamma}\left(\mathbf{r}_{2}, t\right),
\end{aligned}
$$

where, now, the vorticity and vorticity gradient are evaluated at $\mathbf{r}_{1}=\mathbf{r}_{1}(t)$ and $\mathbf{r}_{2}=\mathbf{r}_{2}(t)$. Introducing the Fourier transform of the velocity created by point vortex 2 on point vortex 1:

$$
\mathbf{V}(2 \rightarrow 1, t)=-i \gamma \int \mathbf{k}_{\perp} \hat{u}(k) e^{i \mathbf{k}\left(\mathbf{r}_{1}(t)-\mathbf{r}_{2}(t)\right)} d \mathbf{k}
$$

and making a linear trajectory approximation $\mathbf{r}_{i}(t-\tau) \simeq \mathbf{r}_{i}(t)-\langle\mathbf{V}\rangle\left(\mathbf{r}_{i}, t\right) \tau$, we get

$$
\begin{aligned}
& \frac{\partial \omega_{1}}{\partial t}+\frac{N-1}{N}\langle\mathbf{V}\rangle_{1} \frac{\partial \omega}{\partial \mathbf{r}_{1}}=-\gamma^{2} \frac{\partial}{\partial r_{1}^{\mu}} \int_{0}^{+\infty} d \tau \int d \mathbf{r}_{2} \int d \mathbf{k} d \mathbf{k}^{\prime} k_{\perp}^{\mu} k_{\perp}^{\prime} \hat{u}(k) \hat{u}\left(k^{\prime}\right) e^{-i\left(\mathbf{k}+\mathbf{k}^{\prime}\right)} \boldsymbol{\xi}_{e^{i \mathbf{k}^{\prime} \cdot \mathbf{v} \tau}} \\
& \times\left(\frac{\partial}{\partial r_{1}^{\nu}}-\frac{\partial}{\partial r_{2}^{\nu}}\right) \omega\left(\mathbf{r}_{1}, t\right) \frac{\omega}{\gamma}\left(\mathbf{r}_{2}, t\right)
\end{aligned}
$$

where $\boldsymbol{\xi}=\mathbf{r}_{2}-\mathbf{r}_{1}$ and $\mathbf{v}=\langle\mathbf{V}\rangle\left(\mathbf{r}_{2}, t\right)-\langle\mathbf{V}\rangle\left(\mathbf{r}_{1}, t\right)$. The linear trajectory approximation is clearly not justified for an axisymmetric flow (since the point vortices follow circular trajectories as considered in Sec. 3.2) so it again relies on the (un-controlled) hypothesis that the decorrelation time is extremely short. Integrating on time, we obtain

$$
\begin{array}{r}
\frac{\partial \omega_{1}}{\partial t}+\frac{N-1}{N}\langle\mathbf{V}\rangle_{1} \frac{\partial \omega}{\partial \mathbf{r}_{1}}=-\pi \gamma^{2} \frac{\partial}{\partial r_{1}^{\mu}} \int d \mathbf{r}_{2} \int d \mathbf{k} d \mathbf{k}^{\prime} k_{\perp}^{\mu} k_{\perp}^{\prime} \hat{u}(k) \hat{u}\left(k^{\prime}\right) e^{-i\left(\mathbf{k}+\mathbf{k}^{\prime}\right)} \boldsymbol{\xi}_{\delta}\left(\mathbf{k}^{\prime} \cdot \mathbf{v}\right) \\
\times\left(\frac{\partial}{\partial r_{1}^{\nu}}-\frac{\partial}{\partial r_{2}^{\nu}}\right) \omega\left(\mathbf{r}_{1}, t\right) \frac{\omega}{\gamma}\left(\mathbf{r}_{2}, t\right) .
\end{array}
$$

Now, using the heuristic argument that $\mathbf{k}^{\prime} \cdot \boldsymbol{\xi} \simeq 1$, i.e. $\mathbf{k}^{\prime} \simeq \boldsymbol{\xi} / \xi^{2}$ (due to the exponential $e^{-i \mathbf{k}^{\prime} \boldsymbol{\xi}}$ ), we make the rough substitution $\delta\left(\mathbf{k}^{\prime} \cdot \mathbf{v}\right) \rightarrow \lambda \delta\left(\left(\boldsymbol{\xi} / \xi^{2}\right) \cdot \mathbf{v}\right)=\lambda \xi^{2} \delta(\boldsymbol{\xi} \cdot \mathbf{v})$, where $\lambda$ is a constant of order unity, in Eq. (171). Then, using Eq. (169), we can rewrite Eq. (171) in the form

$$
\begin{array}{r}
\frac{\partial \omega_{1}}{\partial t}+\frac{N-1}{N}\langle\mathbf{V}\rangle_{1} \frac{\partial \omega}{\partial \mathbf{r}_{1}}=\lambda \pi \frac{\partial}{\partial r_{1}^{\mu}} \int d \mathbf{r}_{2} V^{\mu}(2 \rightarrow 1, t) V^{\nu}(2 \rightarrow 1, t) \xi^{2} \delta(\boldsymbol{\xi} \cdot \mathbf{v}) \\
\times\left(\frac{\partial}{\partial r_{1}^{\nu}}-\frac{\partial}{\partial r_{2}^{\nu}}\right) \omega\left(\mathbf{r}_{1}, t\right) \frac{\omega}{\gamma}\left(\mathbf{r}_{2}, t\right) .
\end{array}
$$

Finally, using $\mathbf{V}(2 \rightarrow 1)=-(\gamma / 2 \pi) \boldsymbol{\xi}_{\perp} / \xi^{2}$, we obtain

$$
\frac{\partial \omega_{1}}{\partial t}+\frac{N-1}{N}\langle\mathbf{V}\rangle_{1} \frac{\partial \omega}{\partial \mathbf{r}_{1}}=\frac{\gamma}{8} \frac{\partial}{\partial r_{1}^{\mu}} \int d \mathbf{r}_{2} \frac{\xi^{2} \delta^{\mu \nu}-\xi^{\mu} \xi^{\nu}}{\xi^{2}} \delta(\boldsymbol{\xi} \cdot \mathbf{v})\left(\frac{\partial}{\partial r_{1}^{\nu}}-\frac{\partial}{\partial r_{2}^{\nu}}\right) \omega\left(\mathbf{r}_{1}, t\right) \omega\left(\mathbf{r}_{2}, t\right)
$$

This is Eq. (137) of [23]. The constant $\lambda$ has been determined so that Eq. (173) reproduces at best the exact equations (Eq. (48) and Eq. (135) of [23]) obtained for axisymmetric and unidirectional flows (in particular the expressions of the drift and diffusion in the corresponding Fokker-Planck equation, see [23]). This yields $\lambda=\pi / 2$ which is of order unity as expected. The previous arguments give some "justification" to Eq. (137) of [23] although we again stress that this equation is not exact so it is obtained from un-controlled approximations that are not really justified. 


\section{Drift term for axisymmetric systems}

For an axisymmetric flow, the linearized equation (110) for the perturbation becomes

$$
\frac{\partial \delta \omega}{\partial t}+u_{\theta} \frac{1}{r} \frac{\partial \delta \omega}{\partial \theta}=-V_{r}(P \rightarrow 0) \frac{d \omega}{d r} .
$$

Introducing the angular velocity and the potential of interaction, it can be rewritten

$$
\frac{\partial \delta \omega}{\partial t}+\Omega(r) \frac{\partial \delta \omega}{\partial \theta}=-\frac{\gamma}{r} \frac{\partial u_{O P}}{\partial \theta} \frac{d \omega}{d r}
$$

Taking the Fourier transform of this expression with respect to the angular variable, we obtain

$$
\frac{d \delta \hat{\omega}_{m}}{\partial t}+i m \Omega \delta \hat{\omega}_{m}=-\frac{\gamma}{r} i m \hat{u}_{m}\left(r, r_{P}\right) e^{-i m \theta_{P}} \frac{d \omega}{d r}
$$

Integrating this first order differential equation with respect to time, we find that

$$
\delta \hat{\omega}_{m}(t)=-\int_{0}^{t} d \tau e^{-i m \Omega \tau} \frac{\gamma}{r} i m \hat{u}_{m}\left(r, r_{P}\right) e^{-i m \theta_{P}(t-\tau)} \frac{d \omega}{d r} .
$$

To leading order in $N \rightarrow+\infty$, the test particle is advected by the mean flow so that $\theta_{P}(t-\tau)=$ $\theta_{P}-\Omega_{P} \tau$. Extending the time integral to $+\infty$, we obtain

$$
\delta \hat{\omega}_{m}(t)=-\int_{0}^{+\infty} d \tau e^{-i m\left(\Omega-\Omega_{P}\right) \tau} \frac{\gamma}{r} i m \hat{u}_{m}\left(r, r_{P}\right) e^{-i m \theta_{P}} \frac{d \omega}{d r} .
$$

The integral on time $\tau$ can be easily calculated yielding

$$
\delta \hat{\omega}_{m}(t)=-\pi \delta\left[m\left(\Omega-\Omega_{P}\right)\right] \frac{\gamma}{r} i m \hat{u}_{m}\left(r, r_{P}\right) e^{-i m \theta_{P}} \frac{d \omega}{d r}
$$

The drift velocity experienced by the test particle is

$$
V_{r}^{p o l}=\frac{1}{\gamma} \int V_{r}(1 \rightarrow P) \delta \omega_{1}(t) d \mathbf{r}_{1}
$$

or, more explicitly,

$$
V_{r}^{p o l}=\int_{0}^{+\infty} r_{1} d r_{1} \int_{0}^{2 \pi} d \theta_{1} \frac{1}{r_{P}} \frac{\partial u_{P 1}}{\partial \theta_{P}} \delta \omega_{1} .
$$

Introducing Fourier transforms and substituting Eq. (179) in Eq. (181), this can be rewritten

$$
V_{r}^{p o l}=\frac{2 \pi^{2} \gamma}{r_{P}} \int_{0}^{+\infty} d r_{1} \sum_{m} m^{2} \hat{u}_{m}\left(r_{1}, r_{P}\right)^{2} \delta\left[m\left(\Omega_{1}-\Omega_{P}\right)\right] \frac{d \omega_{1}}{d r_{1}} .
$$

Finally, introducing the notation (47), we obtain

$$
V_{r}^{p o l}=\frac{2 \pi^{2} \gamma}{r_{P}} \int_{0}^{+\infty} d r_{1} \chi\left(r_{1}, r_{P}\right) \delta\left(\Omega_{1}-\Omega_{P}\right) \frac{d \omega_{1}}{d r_{1}} .
$$




\section{References}

[1] U. Frisch, Turbulence (Cambridge University Press 1996).

[2] D. Kivotides, Phys. Rev. Lett. 96, 175301 (2006).

[3] Z. She, E. Jackson, Phys. Rev. Lett. 70, 1255 (1993).

[4] C. Beck, Phys. Rev. Lett. 98, 064502 (2007).

[5] R. Monchaux, F. Ravelet, B. Dubrulle, A. Chiffaudel, F. Daviaux, Phys. Rev. Lett. 96, 124502 (2006).

[6] J. Sommeria, Two-Dimensional Turbulence in: New trends in turbulence, edited by M. Lesieur, A. Yaglom, F. David, Les Houches Summer School 74, 385 (2001).

[7] P. Tabeling, Phys. Rep. 362, 1 (2002).

[8] P.H. Chavanis, Statistical mechanics of two-dimensional vortices and stellar systems in: Dynamics and Thermodynamics of Systems with Long Range Interactions, edited by T. Dauxois, S. Ruffo, E. Arimondo and M. Wilkens, Lect. Not. in Phys. 602, Springer (2002); cond-mat/0212223.

[9] J. McWilliams, J. Fluid. Mech. 146, 21 (1984).

[10] A. Bracco, P.H. Chavanis, A. Provenzale \& E. Spiegel, Phys. Fluids 11, 2280 (1999).

[11] P.S. Marcus, Annu. Rev. Astron. Astrophys. 31, 523 (1993).

[12] J. Pedlosky, Geophysical Fluid Dynamics (Springer-Verlag, Berlin, 1987).

[13] P.K. Newton, The N-Vortex Problem: Analytical Techniques, Applied Mathematical Sciences Vol. 145 (Springer-Verlag, berlin, 2001).

[14] G. Kirchhoff, in Lectures in Mathematical Physics, Mechanics (Teubner, Leipzig, 1877).

[15] Dynamics and Thermodynamics of Systems with Long Range Interactions, edited by T. Dauxois, S. Ruffo, E. Arimondo and M. Wilkens, Lect. Not. in Phys. 602, Springer (2002).

[16] L. Onsager, Nuovo Cimento, Suppl. 6, 279 (1949)

[17] G. Joyce, D. Montgomery, J. Plasma Phys. 10, 107 (1973)

[18] T.S. Lundgren, Y.B. Pointin, J. Stat. Phys. 17, 323 (1977)

[19] E. Caglioti, P.L. Lions, C. Marchioro and M. Pulvirenti, Commun. Math. Phys. 143, 501 (1992).

[20] D. Dubin, T.M. O’Neil, Phys. Rev. Lett. 60, 1286 (1988).

[21] A. Lenard, Ann. Phys. (N.Y.) 10, 390 (1960)

[22] R. Balescu, Phys. Fluids 3, 52 (1960)

[23] P.H. Chavanis, Phys. Rev. E 64, 026309 (2001).

[24] C.R. Willis, R.H. Picard, Phys. Rev. A 9, 1343 (1974).

[25] H. Kandrup, ApJ 244, 316 (1981). 
[26] L.D. Landau, Phys. Z. Sowj. Union 10, 154 (1936).

[27] I. Prigogine, Non-Equilibrium Statistical Mechanics (Interscience, John Wiley, New York, 1962)

[28] S. Chandrasekhar, ApJ 97, 255 (1943).

[29] P.H. Chavanis and M. Lemou, cond-mat/0703023

[30] P.H. Chavanis, Phys. Rev. Lett. 84, 5512 (2000)

[31] D. Lynden-Bell, MNRAS 136, 101 (1967)

[32] J. Miller, Phys. Rev. Lett. 65, 2137 (1990)

[33] R. Robert and J. Sommeria, J. Fluid Mech. 229, 291 (1991)

[34] P.H. Chavanis, J. Sommeria and R. Robert, ApJ 471, 385 (1996)

[35] C.C. Lin, Proc. Natl. Acad. Sci. 27, 570 (1941)

[36] D. Montgomery and G. Joyce, Phys. Fluids 17, 1139 (1974)

[37] P.H. Chavanis, Physica A 361, 55 (2006)

[38] P.H. Chavanis and J. Sommeria, J. Fluid Mech. 356, 259 (1998)

[39] J. Binney, S. Tremaine, Galactic Dynamics (Princeton Series in Astrophysics, 1987)

[40] P.H. Chavanis, Physica A 361, 81 (2006)

[41] F. Bouchet, T. Dauxois, Phys. Rev. E 72, 5103 (2005)

[42] P.H. Chavanis, J. Vatteville, F. Bouchet, Eur. Phys. J. B 46, 61 (2005).

[43] P.H. Chavanis, Physica A 377, 469 (2007)

[44] E.M. Lifshitz, L.P. Pitaevskii, Physical Kinetics (Pergamon Press, Oxford, 1981)

[45] B.B. Kadomtsev, O.P. Pogutse, Phys. Rev. Lett. 25, 1155 (1970)

[46] G. Severne and M. Luwel, Astr. Space Sci. 72, 293 (1980)

[47] P.H. Chavanis, Statistical mechanics of violent relaxation in stellar systems, in: Multiscale Problems in Science and Technology edited by N. Antonic, C.J. van Duijn, W. Jager and A. Rikelic (Springer, Berlin 2002) [astro-ph/0212205]

[48] R. Robert and J. Sommeria, Phys. Rev. Lett. 69, 2776 (1992)

[49] J. Sommeria, C. Staquet, R. Robert, J. Fluid Mech. 233, 661 (1991)

[50] X.P. Huang, C.F. Driscoll, Phys. Rev. Lett. 72, 2187 (1994)

[51] H. Brands, P.H. Chavanis, R. Pasmanter, J. Sommeria, Phys. Fluids 11, 3465 (1999)

[52] P.H. Chavanis, Physica A 365, 102 (2006).

[53] G.F. Carnevale, W.R. Young, J.C. McWilliams, J.B. Weiss, Y. Pomeau, Phys. Rev. Lett. 66, 2735 (1991) 
[54] P.H. Chavanis, Phys. Rev. E 58, R1199 (1998)

[55] P.H. Chavanis, Eur. Phys. J. B 52, 47 (2006)

[56] D. Dubin, D.Z. Jin, Physics Lett. A 284, 112 (2001).

[57] D.A. Schecter, D. Dubin, Phys. Rev. E 13, 1704 (2001).

[58] R. Kawahara, H. Nakanishi, J. Phys. Soc. Jpn 75, 054001 (2006)

[59] R. Kawahara, H. Nakanishi, J. Phys. Soc. Jpn 76, 074001 (2007)

[60] K.S. Fine, A.C. Cass, W.G. Flynn, C.F. Driscoll, Phys. Rev. Lett. 75, 3277 (1995) 